\title{
THE X-RAY STAR FORMATION STORY AS TOLD BY LYMAN BREAK GALAXIES IN THE 4 Ms CDF-S
}

\author{
Antara R. Basu-Zych ${ }^{1}$, Bret D. Lehmer ${ }^{1,2,16}$, Ann E. Hornschemeier ${ }^{1}$, Rychard J. Bouwens ${ }^{3,4}$, Tassos Fragos ${ }^{5}$, \\ Pascal A. Oesch ${ }^{4,17}$, Krzysztof Belczynski ${ }^{6,7}$, W. N. Brandt ${ }^{8,9}$, Vassiliki Kalogera $^{10}$, Bin Luo ${ }^{8,9}$, Neal Miller ${ }^{11}$, \\ James R. Mullaney ${ }^{12}$, Panayiotis Tzanavaris ${ }^{1}$, Yongquan XuE ${ }^{8,9,13}$, and Andreas Zezas ${ }^{5,14,15}$ \\ ${ }^{1}$ NASA Goddard Space Flight Center, Code 662, Greenbelt, MD 20771, USA; antara.r.basu-zych@ nasa.gov \\ 2 Department of Physics and Astronomy, The Johns Hopkins University, 3400 North Charles Street, Baltimore, MD 21218, USA \\ ${ }^{3}$ Leiden Observatory, Leiden University, NL-2300 RA Leiden, The Netherlands \\ ${ }^{4}$ UCO/Lick Observatory, University of California, Santa Cruz, CA 95064, USA \\ ${ }^{5}$ Harvard-Smithsonian Center for Astrophysics, 60 Garden Street, Cambridge, MA 02138, USA \\ ${ }^{6}$ Astronomical Observatory, University of Warsaw, Al. Ujazdowskie 4, 00-478 Warsaw, Poland \\ ${ }^{7}$ Center for Gravitational Wave Astronomy, University of Texas at Brownsville, Brownsville, TX 78520, USA \\ ${ }^{8}$ Department of Astronomy and Astrophysics, Pennsylvania State University, University Park, PA 16802, USA \\ ${ }^{9}$ Institute for Gravitation and the Cosmos, Pennsylvania State University, 525 Davey Lab, University Park, PA 16802, USA \\ ${ }^{10}$ Department of Physics and Astronomy, Northwestern University, 2145 Sheridan Road, Evanston, IL 60208, USA \\ ${ }^{11}$ Department of Astronomy, University of Maryland, College Park, MD 20742, USA \\ ${ }^{12}$ Department of Physics, Durham University, South Road, Durham DH1 3LE, UK \\ ${ }^{13}$ Key Laboratory for Research in Galaxies and Cosmology, Department of Astronomy, University of Science and Technology of China, \\ Chinese Academy of Sciences, Hefei, Anhui 230026, China \\ ${ }^{14}$ Physics Department \& Institute of Theoretical \& Computational Physics, University of Crete, 71003 Heraklion, Crete, Greece \\ ${ }^{15}$ Foundation for Research and Technology-Hellas, 71110 Heraklion, Crete, Greece \\ Received 2012 July 7; accepted 2012 October 10; published 2012 December 14
}

\begin{abstract}
We present results from deep X-ray stacking of $>4000$ high-redshift galaxies from $z \approx 1$ to 8 using the 4 Ms Chandra Deep Field-South data, the deepest X-ray survey of the extragalactic sky to date. The galaxy samples were selected using the Lyman break technique based primarily on recent Hubble Space Telescope ACS and WFC3 observations. Based on such high specific star formation rates (sSFRs): $\log \mathrm{SFR} / M_{*}>-8.7$, we expect that the observed properties of these Lyman break galaxies (LBGs) are dominated by young stellar populations. The X-ray emission in LBGs, eliminating individually detected X-ray sources (potential active galactic nucleus), is expected to be powered by X-ray binaries and hot gas. We find, for the first time, evidence of evolution in the X-ray/SFR relation. Based on X-ray stacking analyses for $z<4$ LBGs (covering $~ 90 \%$ of the universe's history), we find that the $2-10 \mathrm{keV}$ $\mathrm{X}$-ray luminosity evolves weakly with redshift $(z)$ and SFR as $\log L_{\mathrm{X}}=0.93 \log (1+z)+0.65 \log \mathrm{SFR}+39.80$. By comparing our observations with sophisticated X-ray binary population synthesis models, we interpret that the redshift evolution of $L_{\mathrm{X}} / \mathrm{SFR}$ is driven by metallicity evolution in high mass X-ray binaries, likely the dominant population in these high sSFR galaxies. We also compare these models with our observations of X-ray luminosity density (total 2-10 keV luminosity per $\mathrm{Mpc}^{3}$ ) and find excellent agreement. While there are no significant stacked detections at $z \gtrsim 5$, we use our upper limits from $5 \lesssim z \lesssim 8$ LBGs to constrain the supermassive black hole accretion history of the universe around the epoch of reionization.
\end{abstract}

Key words: galaxies: active - galaxies: high-redshift - Galaxy: evolution - stars: evolution - X-rays: binaries

Online-only material: color figures

\section{INTRODUCTION}

Recently, Chandra completed the deepest X-ray survey to date: 4 Ms in the Chandra Deep Field-South (CDF-S; Xue et al. 2011, hereafter X11). The last leap forward in X-ray survey depth occurred approximately a decade ago as deep surveys moved from $1 \mathrm{Ms}$ to $2 \mathrm{Ms}$ coverage (e.g., Alexander et al. 2003). Such deep X-ray surveys are crucial to studying the high-energy emission from distant star-forming galaxies, which overall are an extremely X-ray faint population compared to the more luminous active galactic nucleus (AGN) population that dominates the number counts at bright fluxes (e.g., see Lehmer et al. 2012). Notably, since the profusion of papers several years ago on high redshift $(z>2) \mathrm{X}$-ray studies of star-forming galaxies (i.e., Reddy \& Steidel 2004; Laird et al. 2005, 2006; Lehmer et al. 2005b), there have been significant improvements in the identification of new high-redshift galaxy

\footnotetext{
${ }^{16}$ Einstein Fellow.

${ }^{17}$ Hubble Fellow.
}

samples, selected using the Lyman break technique, from $z=$ 1.5-8 (Beckwith et al. 2006; Bouwens et al. 2007, 2008, 2010; Reddy \& Steidel 2009; Oesch et al. 2010b; Hathi et al. 2010; Bunker et al. 2010). Thus, it is timely to study the evolution of X-ray properties in star-forming galaxies with these updated data sets.

The Lyman break technique efficiently selects distant starforming galaxies based on a strong spectral break at $912 \AA$, caused by Lyman series absorption of neutral hydrogen in the intergalactic medium; such galaxies are referred to as Lyman break galaxies (LBGs; Steidel \& Hamilton 1992, 1993; Steidel et al. 1995, 2000). The color selection applied in searching for LBGs requires bright rest-frame UV continua and blue colors, therefore selecting galaxies with recent star formation and stellar populations dominated by young stars. LBGs have greatly impacted investigations of galaxy evolution. For example, significant effort has been devoted to studying the cosmic star formation history and particularly in measuring the peak (and decline at $z>3-4$ ) of the global star formation 
rate (SFR) density using LBGs (Madau et al. 1996; Bouwens et al. 2006, 2009, 2011). However, dust attenuation in UVselected galaxies poses challenges to measuring the total SFR in these galaxies. Other samples of galaxies (e.g., submillimeter selected; Blain et al. 1999) have been studied to provide additional constraints on the SFR density.

$\mathrm{X}$-rays offer another way to explore the dust-unobscured cosmic star formation history. X-rays in "normal galaxies" (i.e., not dominated by AGNs) mainly originate from accreting $\mathrm{X}$-ray binaries (XRBs, including ultraluminous X-ray sources). The 2-10 keV emission in normal local galaxies scales with SFR (Ranalli et al. 2003; Persic \& Rephaeli 2007; Mineo et al. 2012). However, a number of other sources can contribute to the X-ray emission in normal galaxies: supernovae and their remnants, and hot gas from starburst-driven winds and outflows (see, e.g., review by Fabbiano 1989). Low-luminosity AGN activity may also contribute to the X-ray luminosity in normal galaxies.

Within the XRB population, high mass X-ray binaries (HMXBs) are short lived, tracing recent star formation activity (on timescales $\sim 10^{6}-10^{7} \mathrm{yr}$ ), while low mass X-ray binaries $(\mathrm{LMXBs}$ ) trace older stellar populations (for timescales $>10^{8}-10^{8} \mathrm{yr}$ ) and the $2-10 \mathrm{keV}$ X-ray luminosity scales with the stellar mass $\left(M_{*}\right)$ of the galaxy. Assuming the following analytic parameterization for local (within a distance of $60 \mathrm{Mpc}$ ) normal galaxies:

$$
\begin{aligned}
L_{\mathrm{X}, 2-10 \mathrm{keV}} & =L_{\mathrm{X}}(\mathrm{LMXB})+L_{\mathrm{X}}(\mathrm{HMXB}) \\
& =\alpha M_{*}+\beta \mathrm{SFR} .
\end{aligned}
$$

Lehmer et al. (2010) measured constants of $\alpha=(9.05 \pm 0.37) \times$ $10^{28} \mathrm{erg} \mathrm{s}^{-1} M_{\odot}^{-1}$ and $\beta=(1.62 \pm 0.22) \times 10^{39} \mathrm{erg} \mathrm{s}^{-1}$ $\left(M_{\odot} \mathrm{yr}^{-1}\right)^{-1}$ (see also Colbert et al. 2004). As a relatively dustinsensitive probe of past and present SFR, the average X-ray properties of star-forming galaxies give an independent measurement of the cosmic star formation history. In addition, the wide range of evolutionary timescales, SFRs, and metallicities probed by deep surveys to high redshift offers constraints on binary evolution theories (e.g., Fragos et al. 2012, hereafter, referred to as F12).

Several studies have shown the utility of stacking deep $\mathrm{X}$-ray data at the optically determined locations of star-forming galaxies to measure the average $\mathrm{X}$-ray properties of distant LBG populations that are individually undetected. Averaging the $\mathrm{X}$-ray counts at the known positions of the targets enhances the signal-to-noise ratio $(\mathrm{S} / \mathrm{N})$ for source populations with fluxes below the detection limit. Through stacking, the relationship between X-rays and other galaxy properties (e.g., SFR, stellar mass, dust attenuation) has been investigated for low-redshift galaxies $(z<1.4$; Ptak et al. 2001; Hornschemeier et al. 2002; Laird et al. 2005; Lehmer et al. 2007, 2008; Watson et al. 2009; Symeonidis et al. 2011), intermediate-redshift LBGs $(1.5<z<3$; Brandt et al. 2001; Reddy \& Steidel 2004), and distant LBGs $(3<z<6$; Brandt et al. 2001; Laird et al. 2006; Lehmer et al. 2005b; Cowie et al. 2012). These stacking studies highlight the use of X-rays from normal galaxies to study the star formation history of the universe. For example, Symeonidis et al. (2011) correlate X-ray emission with infrared (IR) emission for galaxies at $0<z<2$ and show that X-ray emission can be calibrated as a useful star formation indicator to measure the global SFR density.

In this paper, we perform stacking analyses of LBGs, using the deepest X-ray observations to date (4 Ms Chandra observations of CDF-S; X11), adding newly discovered LBGs and covering the broad redshift range, $1.5 \lesssim z \lesssim 8$ (i.e., corresponding to when the age of the universe was $4.5-0.6 \mathrm{Gyr}$ ). Cowie et al. (2012) have completed a study of X-ray emission in this sample to find that X-ray emission from $z=0-8$ LBGs is consistent with normal galaxy populations, provided that a reasonable range of dust attenuation factors $(\sim 3-5)$ are present. However, Cowie et al. (2012) did not attempt to correct for dust attenuation and search for low levels of evolution in the X-ray emission. For the first time, we measure an empirical law that relates X-ray luminosity, SFR and redshift $(z)$ and compare our results with XRB evolution models (see F12).

Our paper is organized as follows: we introduce the samples and describe our stacking analysis techniques in Section 2; we discuss the individually detected X-ray sources in Section 3.1 and the stacking results for $z \lesssim 4$ and $z \gtrsim 5$ in Sections 3.2-3.4; finally, we offer our conclusions in Section 4. The Galactic column densities are $8.8 \times 10^{19} \mathrm{~cm}^{-2}$ for the E-CDF-S (Stark et al. 1992). All of the X-ray fluxes and luminosities quoted throughout this paper have been corrected for Galactic absorption. We assume the standard $\Lambda \mathrm{CDM}$ cosmology: $\Omega_{M}=0.3, \Omega_{\Lambda}=0.7$, and $H_{0}=70 \mathrm{~km} \mathrm{~s}^{-1} \mathrm{Mpc}^{-1}$, and the initial mass function (IMF) described by Kroupa (2001).

\section{DATA AND ANALYSIS}

For our analysis of the X-ray properties of LBGs, we use the publicly available $4 \mathrm{Ms}$ CDF-S catalog and data products from X11. ${ }^{18}$

We use $1.5 \lesssim z \lesssim 8$ LBG samples compiled from several catalogs (see Table 1): $z \approx 1.5,1.9$, and 2.5 from Oesch et al. $(2010 \mathrm{a}), z \approx 3$ from Lee et al. (2006), $z \approx 3.8,5.0$, and 5.9 from Bouwens et al. (2007), and $z \approx 6.8$ and 8.0 from Bouwens et al. (2011). We include additional $z \approx 5-8$ LBGs (Bouwens et al. 2012a; Oesch et al. 2012) that have been identified in the CDF-S field from data obtained in the CANDELS survey (Grogin et al. 2011; Koekemoer et al. 2011). We refer readers to these papers for details about the LBG selection; however, the basic technique for selecting these galaxies involves using photometric color selection to isolate galaxies with relatively flat spectra that drop out of the bluest filter, while avoiding the expected color tracks of nearby interlopers (stars, low-redshift elliptical galaxies).

The areal coverages of the LBG samples are shown in Figure 1 on top of the Chandra $0.5-8 \mathrm{keV}$ full-band image. The blue region marks the LBGs selected from the Hubble Space Telescope (HST) Wide Field Camera 3 (WFC3) Early Release Science data, i.e., galaxies dropping out of the F225W $(z \approx 1.5 ; 48$ galaxies $), \mathrm{F} 275 \mathrm{~W}(z \approx 1.9 ; 91$ galaxies $)$, and $\mathrm{F} 336 \mathrm{~W}(z \approx 2.5 ; 359$ galaxies $)$ filters. The green region contains the $361 z \approx 3.0$ LBG sample, selected as U-dropouts, based on ground-based $U$-band observations taken on the $4 \mathrm{~m}$ telescope at Cerro Tololo Inter-American Observatory (CTIO) of the Great Observatories Origins Deep Survey-South (GOODS-S) field. The orange contour bounds the samples of $2098 \mathrm{~B}_{435^{-}}$ $(z \approx 3.8), 445 V_{606^{-}}(z \approx 5.0)$, and $181 i_{775^{-}}(z \approx 5.9)$ dropouts selected using the Advanced Camera for Surveys (ACS) instrument aboard HST, which includes the GOODS-S field and two additional regions from parallel fields for NICMOS Ultra-deep Field (PI: Roger Thompson; Thompson et al. 2005). The red dashed regions mark the locations of $73 z \approx 7$ and 60 18 Data products are available online at http://www2.astro.psu.edu/ niel/cdfs/
cdfs-chandra.html. 
Table 1

Lyman Break Galaxy Samples for Deep Chandra X-Ray Study

\begin{tabular}{|c|c|c|c|c|c|c|c|}
\hline$z$ & Dropout Band & Instrument & $\begin{array}{c}\text { Area } \\
\left(\operatorname{arcmin}^{2}\right)\end{array}$ & $N_{\text {LBGs }}$ & $\begin{array}{c}\left\langle m_{\mathrm{UV}}\right\rangle^{\mathrm{a}} \\
(\mathrm{mag})\end{array}$ & $\begin{array}{c}\left\langle\mathrm{SFR}_{\mathrm{UV}, \text { corr }}\right\rangle^{\mathrm{b}} \\
\left(M_{\odot} \mathrm{yr}^{-1}\right)\end{array}$ & Reference \\
\hline $1.5 \pm 0.4$ & F225W (2372 ̊) & $H S T / \mathrm{WFC} 3$ & 50 & 48 & 24.5 & 13 & Oesch et al. $(2010 a)^{c}$ \\
\hline $1.9 \pm 0.4$ & F275W (2710 A $)$ & $H S T / \mathrm{WFC} 3$ & 50 & 91 & 24.8 & 30 & Oesch et al. $(2010 a)^{c}$ \\
\hline $2.5 \pm 0.6$ & F336W (3355 Å) & $H S T / \mathrm{WFC} 3$ & 50 & 359 & 25.4 & 17 & Oesch et al. $(2010 a)^{c}$ \\
\hline $3.0 \pm 0.2$ & $\mathrm{U}(3570 \AA)$ & $\mathrm{CTIO}+H S T / \mathrm{ACS}$ & 160 & 361 & 25.3 & 33 & Lee et al. $(2006)^{c}$ \\
\hline $3.8 \pm 0.3$ & $B_{435}(4317 \AA)$ & $H S T / \mathrm{ACS}$ & 196 & 2098 & 26.5 & 8 & Bouwens et al. (2007) \\
\hline \multirow[t]{2}{*}{$5.0 \pm 0.3$} & $V_{606}(5918 \AA ⿻)$ & $H S T / \mathrm{ACS}$ & 196 & 445 & 26.5 & 10 & Bouwens et al. (2007) \\
\hline & & HST/WFC3 (CANDELS) & 105 & $768(700)^{\mathrm{d}}$ & 26.8 & 10 & Bouwens et al. (2012a) \\
\hline \multirow[t]{2}{*}{$5.9 \pm 0.3$} & $i_{775}(7693 \AA)$ & $H S T / \mathrm{ACS}$ & 196 & 181 & 27.0 & 5 & Bouwens et al. (2007) \\
\hline & & HST/WFC3 (CANDELS) & 105 & $218(208)^{\mathrm{d}}$ & 27.0 & 8 & Bouwens et al. (2012a) \\
\hline \multirow[t]{2}{*}{$6.8 \pm 0.4$} & $z_{850}(9055 \AA)$ & $H S T / \mathrm{ACS}$ & 150 & 73 & 27.9 & 4 & Bouwens et al. (2011) \\
\hline & & HST/WFC3 (CANDELS) & 105 & $41(31)^{\mathrm{d}}$ & 26.7 & 11 & Bouwens et al. (2012a) \\
\hline \multirow[t]{2}{*}{$8.0 \pm 0.5$} & $Y_{105}(1.055 \mu \mathrm{m})$ & $H S T / \mathrm{WFC} 3$ & 150 & 60 & 28.0 & 2 & Bouwens et al. (2011) \\
\hline & & HST/WFC3 (CANDELS) & 105 & $24(22)^{\mathrm{d}}$ & 27.0 & 5 & Bouwens et al. (2012a) \\
\hline
\end{tabular}

Notes.

${ }^{a}$ Mean apparent rest-frame UV magnitude.

b Mean dust-corrected UV SFR.

${ }^{c}$ Catalog of sources was acquired via private communication.

d The total number of LBGs in the dropout sample, with the number of unique (not included in ACS sample) LBGs in parentheses.

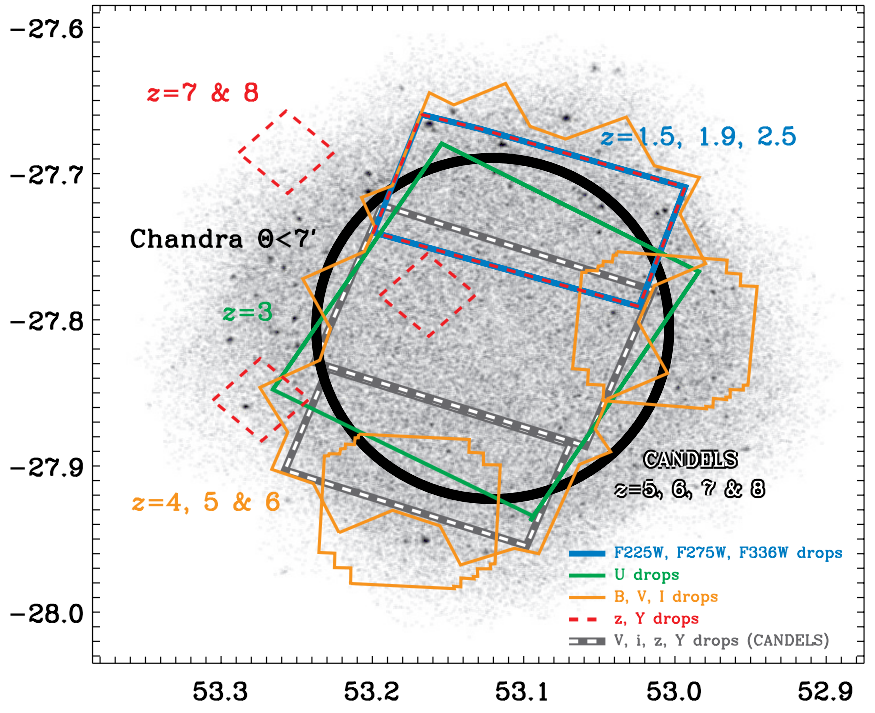

Figure 1. We show the areal coverage of the different LBG samples, overlaid on the 4 Ms Chandra full band (0.5-8 keV) image of CDF-S. The F225W$(z=1.5)$, F275W- $(z=1.9)$, and F336W- $(z=2.5)$ dropouts fall within the blue region; U-dropouts $(z=3)$ lie within the green region; $B_{435^{-}}(z=3.8)$, $V_{606^{-}}(z=5.0)$, and $i_{775^{-}}(z=5.9)$ dropouts are located within the orange contour; $z_{850^{-}}(z=6.9)$ and $Y_{105^{-}}(z=8.0)$ dropouts are bounded by the red dashed lines. Additional $V_{606^{-}}, i_{775^{-}}, z_{850^{-}}$, and $Y_{105^{-}}$-dropouts reside in the CANDELS fields (shown in gray and white dashed lines). The thick black line shows the Chandra area with off-axis angle $<7^{\prime}$.

$z \approx 8$ LBGs, selected from dropping out of the $z_{850}$ and $Y_{105}$ filters using HST ACS and WFC3 infrared data. The CANDELS fields, shown with gray and white dashed lines (CANDELSDeep in upper region; CANDELS-Wide field in lower), add 700 $V_{606^{-}}, 208 i_{775^{-}}, 31 z_{850^{-}}$, and $22 Y_{105^{-d r o p o u t s}}$ This information is summarized in Table 1.

Contamination from various sources (e.g., low-mass stars, AGNs, low-redshift galaxies, transient sources, photometric scatter, and spurious sources) in the LBG sample is expected to range from $3 \%$ to $10 \%$, and is discussed in more detail in Bouwens et al. (2007, 2011).

\subsection{LBG Samples: Determining UV-based Dust-corrected SFRs}

In order to study the relationship between X-ray luminosity and SFRs in LBGs, we estimate their SFRs based on rest-frame UV properties. We use the relation given in Equation (1) of Bell et al. (2005) to convert rest-frame UV luminosities into UV-derived SFRs, given by the right side of this equation:

$$
\operatorname{SFR}\left(M_{\odot} \mathrm{yr}^{-1}\right)=9.8 \times 10^{-11}\left(L_{\mathrm{IR}}+2.2 L_{\mathrm{UV}}\right) / L_{\odot},
$$

where $L_{\mathrm{UV}}$ is the UV luminosity $\left(=\nu 1_{v, 2800}\right.$, where $v$ is the frequency, and $l_{v, 2800}$ is the monochromatic luminosity measured at rest-frame $2800 \AA$ ) and $L_{\odot}$ is solar luminosity $\left(L_{\odot}=3.84 \times 10^{33} \mathrm{erg} \mathrm{s}^{-1}\right)$. Based on the available LBG catalogs, we note that the given UV magnitudes correspond to rest-frame $1500 \AA$ for some galaxy samples $(z \approx 1.5,1.9$, and 2.5) and to $2800 \AA$ for the other samples. Since the LBG galaxy spectrum $\left(L_{v}\right)$ is expected to be very flat in this spectral region there are negligible corrections to the SFR based on these differences (Kennicutt 1998).

We derive dust-corrected SFRs following the prescription given in Bouwens et al. (2012b). Using the measured UVcontinuum slopes $(\beta)$ in $z \approx 2-7$ LBGs (Bouwens et al. 2009, $2012 b$ ), Bouwens et al. (2012b) provide a conversion of the observed UV absolute magnitude and redshift into an estimated $\beta$. We apply the relation between $\beta$ and FUV dust attenuation from Meurer et al. (1999) to estimate the extinction factor for each galaxy, which also takes into account the typical scatter of $\beta$ values $(\sim 0.36)$ at an observed UV magnitude and redshift. These extinction factors range from 3 to 6 for the $z<3$ LBGs and are $<2$ at $z>3$, with typical uncertainties of $\sim 0.3$ (see Table 4). As an additional check on the dust-corrected SFRs, we compare the average dust-corrected UV SFR with radio- and UV+IR-derived SFRs for each redshift sample in Section 2.5.

We show the SFR distributions of LBG samples in Figure 2. We divide our LBG samples into separate SFR bins (marked by the dotted lines): $\mathrm{SFR} /\left(M_{\odot} \mathrm{yr}^{-1}\right)=1-5$ (low SFR), $\mathrm{SFR} /\left(M_{\odot} \mathrm{yr}^{-1}\right)=5-15($ medium SFR $)$, and SFR $/\left(M_{\odot} \mathrm{yr}^{-1}\right)=$ 15-100 (high SFR) with the numbers of galaxies that fall in each bin labeled. This choice of SFR binning was selected to match 
Table 2

Stellar Mass Properties for $z<6$ Lyman Break Galaxies

\begin{tabular}{|c|c|c|c|c|c|c|c|c|}
\hline$z$ & $N_{\text {match }^{\mathrm{a}}}$ & $N_{\text {tot }}^{\mathrm{b}}$ & $\begin{array}{c}\left\langle\log M_{*}\right\rangle \\
\left(M_{\odot}\right)\end{array}$ & $\begin{array}{c}\text { Med }\left(\log M_{*}\right)^{\mathrm{c}} \\
\left(M_{\odot}\right)\end{array}$ & $\begin{array}{c}\langle\log \mathrm{SFR}\rangle \\
\left(M_{\odot} \mathrm{yr}^{-1}\right)\end{array}$ & $\begin{array}{c}\text { Med }(\log \mathrm{SFR})^{\mathrm{c}} \\
\left(M_{\odot} \mathrm{yr}^{-1}\right)\end{array}$ & $\begin{array}{c}\langle\log \mathrm{sSFR}\rangle \\
\left(\mathrm{yr}^{-1}\right)\end{array}$ & $\begin{array}{c}\text { Med }(\log \mathrm{sSFR})^{\mathrm{c}} \\
\left(\mathrm{yr}^{-1}\right)\end{array}$ \\
\hline \multicolumn{9}{|c|}{ All SFRs } \\
\hline 1.5 & 42 & 47 & $9.6 \pm 0.6$ & 9.5 & $1.0 \pm 0.3$ & 1.0 & $-8.6 \pm 0.6$ & -8.4 \\
\hline 1.9 & 59 & 91 & $9.5 \pm 0.5$ & 9.4 & $1.4 \pm 0.3$ & 1.4 & $-8.1 \pm 0.5$ & -8.1 \\
\hline 2.5 & 127 & 358 & $9.4 \pm 0.7$ & 9.4 & $1.2 \pm 0.4$ & 1.2 & $-8.2 \pm 0.6$ & -8.2 \\
\hline 3.0 & 156 & 360 & $9.5 \pm 0.6$ & 9.5 & $1.2 \pm 0.3$ & 1.1 & $-8.3 \pm 0.5$ & -8.3 \\
\hline 3.8 & 982 & 1847 & $8.9 \pm 0.6$ & 8.9 & $0.8 \pm 0.4$ & 0.7 & $-8.1 \pm 0.5$ & -8.1 \\
\hline 5.0 & 414 & 874 & $8.9 \pm 0.8$ & 8.9 & $0.8 \pm 0.3$ & 0.8 & $-8.1 \pm 0.7$ & -8.1 \\
\hline 5.9 & 136 & 292 & $8.9 \pm 0.9$ & 9.0 & $0.7 \pm 0.3$ & 0.7 & $-8.2 \pm 0.8$ & -8.3 \\
\hline 6.8 & 4 & 65 & $9.5 \pm 0.6$ & 9.9 & $1.5 \pm 0.2$ & 1.5 & $-8.0 \pm 0.4$ & -8.0 \\
\hline \multicolumn{9}{|c|}{ Medium SFRs: SFR $=5-15 M_{\odot} \mathrm{yr}^{-1}$} \\
\hline 1.5 & 28 & 31 & $9.6 \pm 0.6$ & 9.6 & $1.0 \pm 0.1$ & 1.0 & $-8.7 \pm 0.7$ & -8.5 \\
\hline 1.9 & 17 & 28 & $9.6 \pm 0.8$ & 9.3 & $1.1 \pm 0.1$ & 1.1 & $-8.5 \pm 0.8$ & -8.3 \\
\hline 2.5 & 49 & 167 & $9.2 \pm 0.7$ & 9.2 & $0.9 \pm 0.1$ & 0.9 & $-8.3 \pm 0.7$ & -8.2 \\
\hline 3.0 & 89 & 224 & $9.3 \pm 0.5$ & 9.3 & $1.0 \pm 0.1$ & 1.0 & $-8.3 \pm 0.5$ & -8.3 \\
\hline 3.8 & 357 & 580 & $9.0 \pm 0.5$ & 9.1 & $0.9 \pm 0.1$ & 0.9 & $-8.1 \pm 0.5$ & -8.1 \\
\hline 5.0 & 196 & 348 & $9.0 \pm 0.8$ & 9.0 & $0.9 \pm 0.1$ & 0.9 & $-8.1 \pm 0.7$ & -8.1 \\
\hline 5.9 & 53 & 80 & $8.9 \pm 0.9$ & 9.0 & $0.8 \pm 0.1$ & 0.8 & $-8.0 \pm 0.8$ & -8.0 \\
\hline \multicolumn{9}{|c|}{ High SFRs: SFRs $=15-100 M_{\odot} \mathrm{yr}^{-1}$} \\
\hline 1.5 & 8 & 9 & $9.8 \pm 0.5$ & 9.8 & $1.5 \pm 0.1$ & 1.5 & $-8.3 \pm 0.6$ & -8.1 \\
\hline 1.9 & 40 & 61 & $9.5 \pm 0.4$ & 9.5 & $1.5 \pm 0.2$ & 1.5 & $-8.0 \pm 0.3$ & -8.0 \\
\hline 2.5 & 56 & 105 & $9.7 \pm 0.5$ & 9.7 & $1.5 \pm 0.2$ & 1.5 & $-8.2 \pm 0.5$ & -8.1 \\
\hline 3.0 & 64 & 119 & $9.8 \pm 0.5$ & 9.7 & $1.5 \pm 0.2$ & 1.4 & $-8.3 \pm 0.5$ & -8.2 \\
\hline 3.8 & 156 & 231 & $9.5 \pm 0.5$ & 9.5 & $1.4 \pm 0.2$ & 1.4 & $-8.1 \pm 0.5$ & -8.1 \\
\hline 5.0 & 57 & 112 & $9.5 \pm 0.7$ & 9.5 & $1.4 \pm 0.2$ & 1.3 & $-8.1 \pm 0.7$ & -8.2 \\
\hline 5.9 & 10 & 14 & $9.9 \pm 0.4$ & 10.0 & $1.3 \pm 0.2$ & 1.4 & $-8.6 \pm 0.3$ & -8.5 \\
\hline 6.8 & 4 & 9 & $9.5 \pm 0.6$ & 9.9 & $1.5 \pm 0.2$ & 1.5 & $-8.0 \pm 0.4$ & -8.0 \\
\hline
\end{tabular}

Notes.

a The number of matches with Xue et al. (2012) with $|\Delta z|<0.5$.

b The total number of LBGs within Xue et al. (2012) footprint.

c Median values.

the SFR binning at low redshifts (L08; Lehmer et al. 2010) for direct comparison. The dark gray arrows mark the mean for each SFR bin and the light gray marks the expect mean, calculated using the UV luminosity function (UVLF; the form is described by the parameters given in the papers listed in Table 1 for each LBG sample) to predict the number of galaxies beyond the observed limit. The $z \lesssim 3$ medium SFR samples appear to suffer from incompleteness, in that the observed mean SFRs are overestimated compared to the true mean for LBGs. We discuss the implications of this incompleteness on our results in Sections 3.2 and 3.3.

To compare stellar masses, $M_{*}$, for these LBGs, we match to the Xue et al. (2012) catalog, which uses the available (12 bands) photometric data to estimate stellar masses. We only consider matches where the redshifts (between our sample and the Xue et al. 2012 catalog) agree within $|\Delta z|<0.5$. The number of matches decreases for fainter and higher redshift objects. Only four of the $z>6$ LBGs were matched to sources in the Xue et al. (2012) catalog; since the Xue et al. (2012) catalog has a $5 \sigma z_{850}$-band limiting magnitude of 28.1 , it is likely that most of our highest redshift LBGs are undetected in this filter. The Xue et al. (2012) catalog gives the most likely redshift, but it is possible that the second most likely redshift would match the LBG selection redshift (e.g., see Section 3.1 about XID = 371). While the $|\Delta z|<0.5$ criterion diminishes the sample size significantly, the average and median $M_{*}$ offer valuable information about the samples: $\left\langle\log M_{*}\right\rangle=8.9-9.6$ for the full sample, 8.9-9.6 for the medium SFR sample, and 9.5-9.8 for the high SFR sample. The specific SFRs (sSFRs $\equiv \mathrm{SFR} / M_{*}$ ) are $\langle\log \mathrm{sSFR}\rangle=-8.7$ to -8.0 . The median and mean values for $M_{*}, \mathrm{SFR}$, and sSFR are given for each sub-sample (redshift and SFR range) in Table 2. Given the high sSFRs for these galaxies, the stellar populations in these galaxies are likely relatively young, exhibiting high present to past SFRs.

\subsection{LBG Samples: Individually Detected X-Ray Sources}

Since our primary goal is to study the X-ray emission arising from star formation in these high-redshift LBGs, we avoid including AGNs in our X-ray stacking analyses. We assume that sources that have been individually detected at these redshifts (and therefore with $L_{\mathrm{X}}>10^{42} \mathrm{erg} \mathrm{s}^{-1}$ ) must be dominated by emission from AGNs.

X11 have matched the X-ray-detected sources to optical, infrared, and radio catalogs using probabilistic matching, which estimates the false match statistics by applying a Monte Carlo technique (see also Broos et al. 2011) and accounts for positional uncertainties (which vary across the Chandra field with offaxis angle). X11 found an offset between the optical/infrared positions and radio positions (the X-ray positions are fixed to the radio frame): R.A.X,radio $=\mathrm{R}$.A. opt, $\mathrm{IR}+0^{\prime}$. 175 and Decl. $_{\text {X,radio }}=$ Decl. $_{\text {opt }, \mathrm{IR}}-0{ }^{\prime} .284$. The coordinates ( shifted to the radio/X-ray system) for the best matched GOODS-S or GEMS sources are provided, which we match to our LBG positions within 0'.5. We find $20 \mathrm{X}$-ray-detected LBGs (which are likely AGNs) and tabulate their properties in Table 3. 


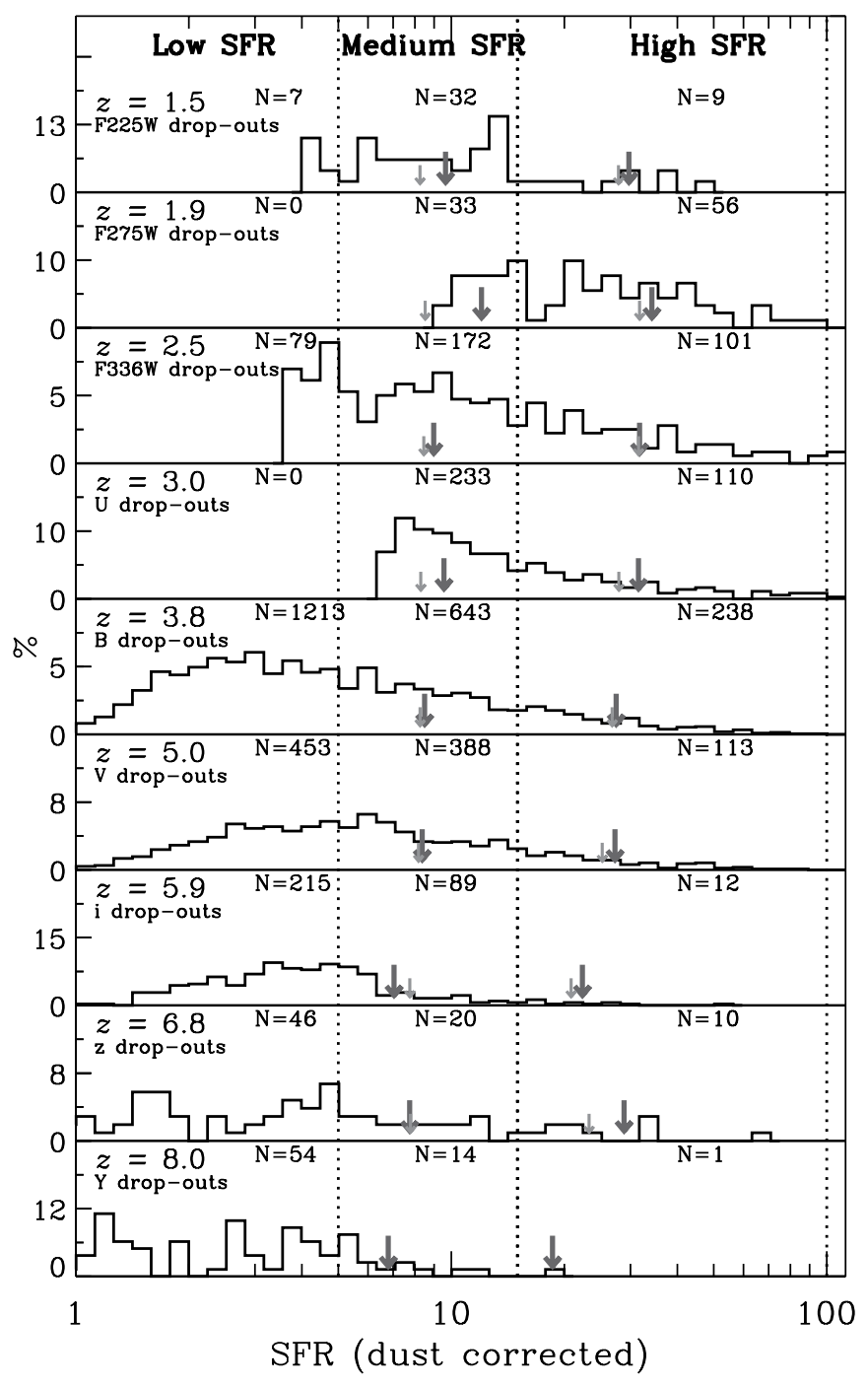

Figure 2. We show the dust-corrected UV-derived SFR distributions of the LBG samples used in this study. The dotted lines mark our SFR cuts for two separate stacking bins: $\operatorname{SFR} /\left(M_{\odot} \mathrm{yr}^{-1}\right)=5-15$ and SFR $/\left(M_{\odot} \mathrm{yr}^{-1}\right)=15-100$ with the numbers of galaxies that fall in each bin labeled. The arrows mark the observed mean (dark gray) for the different SFR bins compared to the expected mean (light gray; based on the UVLF). The lowest SFR bins do not yield any stacked detections and suffer from incompleteness; therefore, they are not discussed further.

In this table, the redshifts based on the LBG sample selection (z) are listed along with the redshifts from the X11 catalog, based on spectroscopic $(s)$ or other photometric data $(p)$. The effective photon index $(\Gamma)$ was determined for all sources with $>200$ full band $(0.5-8 \mathrm{keV})$ counts using basic spectral fitting (described in X11) and assuming an absorbed power law. The last column in this table includes notes about previously detected and studied sources from CDF-S 1 Ms (L05), E-CDF-S catalog (Lehmer et al. 2005a, hereafter L05a), and radio (R) detections based on our Very Large Array (VLA) data analysis (see Section 2.5). These sources are discussed in more detail in Section 3.1.

\subsection{X-Ray Stacking of Individually Undetected LBGs}

While even 4 Ms of exposure time is not sufficient for detecting X-rays from individual star-forming galaxies at $z>$ 1 (with the possible exception of submillimeter galaxies; Alexander et al. 2005; Laird et al. 2010), the X-ray emission from large numbers of galaxies can be stacked to provide the average X-ray properties of the stacked sources. We follow the stacking technique outlined in Lehmer et al. (2008, hereafter, L08) to stack LBGs in bins of SFRs (shown in Figure 2) and $z$.

First, we select sources that have no X-ray detections in the catalog from X11 within twice the $99 \%$ encircled energy fraction (EEF) radius (note that this value changes with off-axis angle) in order to avoid contamination from the Chandra point-spread function (PSF) from detected sources. We only include sources with off-axis angles $<7^{\prime}$ from the central pointing (thick black circle in Figure 1) because the increasing PSF at large off-axis angles degrades the X-ray sensitivity significantly. These two criteria eliminate 856 and 837 LBGs, respectively, from our original sample of LBGs.

Recent stacking results of $z>6$ LBGs by Treister et al. (2010) have generated discussion about proper background subtraction in order to avoid artificial stacked detections (e.g., by setting the clipping threshold of the background pixels too low; see Cowie et al. 2012; Willott 2011). We refer readers to L05a and L08 for detailed descriptions about our background subtraction technique using background maps, which do not include any clipping of photons. Briefly, these background maps were created by filling the regions near detected sources (twice the $90 \%$ PSF EEF radius, $r_{90}$ ) with noise, which is generated based on the probability distribution of counts in the local background (within an annulus with radii between 2 and $4 \times r_{90}$ ).

Throughout the remainder of this paper, X-ray luminosities refer to the rest-frame hard $(2-10 \mathrm{keV})$ energy band. These luminosities are $k$-corrected from the observed soft band $(0.5-2 \mathrm{keV})$ since this band is closer to the rest-frame $2-10 \mathrm{keV}$ band, and therefore the $k$-correction is smaller and less affected by the assumed spectral shape (i.e., photon index, $\Gamma$ ). For example, at the median redshift of our LBG samples $(z \approx 3)$ the observed $0.5-2 \mathrm{keV}$ band corresponds to rest-frame $2-8 \mathrm{keV}$. The $k$-correction can be calculated as follows:

$$
\begin{aligned}
k_{\mathrm{corr}} & =\frac{E_{\mathrm{out}, 2}^{(2-\Gamma)}-E_{\mathrm{out}, 1}^{(2-\Gamma)}}{E_{\mathrm{in}, 2}^{(2-\Gamma)}-E_{\mathrm{in}, 1}^{(2-\Gamma)}}(1+z)^{(\Gamma-2.0)} \\
& =2.472(1+z)^{-0.3}
\end{aligned}
$$

using $\Gamma=1.7$, based on the expected photon index for XRBs (Ptak et al. 1999) and in the input and output energies: $E_{\text {out }, 1}=2$ $\mathrm{keV}, E_{\text {out }, 2}=10 \mathrm{keV}, E_{\text {in }, 1}=0.5 \mathrm{keV}$, and $E_{\text {in, } 1}=2 \mathrm{keV} .{ }^{19}$

We consider two sources of error in our stacked measurements. The first, $\sigma_{\mathrm{p}}$, is Poisson noise and calculated simply as $\sqrt{B}$, where $B$ represents background counts. This is the error that we use to determine the $\mathrm{S} / \mathrm{N}$. In addition, we also calculate the errors from bootstrapping $\left(\sigma_{\text {boot }}\right)$, which measure how the contribution of individual sources may affect the average value. To determine our bootstrapping errors, we randomly resample the LBGs for each stacking bin a large number of times (5000) and repeat our stacking analysis on these samples. The random resampling will duplicate some values while eliminating others each iteration to statistically quantify the effect of individual sources on the stack ( $\sim 37 \%$ of the values are replaced by duplicated values; see Efron 1982). The bootstrapped errors refer to the standard deviation of the stacked values from this random

\footnotetext{
19 We note that we attempted to stack in the observed $2-8 \mathrm{keV}$ bandpass, however, we did not obtain $>2 \sigma$ detections in any case. Ideally, we could use the $2-8 \mathrm{keV} / 0.5-2 \mathrm{keV}$ stacked band ratios as proxies for the spectral slopes of our stacks. Unfortunately, the upper limits of the band ratios are not well constrained $\left(\Gamma_{\text {eff }}>0.3-0.7\right)$ for all cases. Therefore, we limit further discussion to results based on stacking the observed soft $0.5-2 \mathrm{keV}$ band.
} 
Table 3

Individually Detected X-Ray Sources Associated with Lyman Break Galaxies

\begin{tabular}{|c|c|c|c|c|c|c|c|c|c|c|}
\hline XID & R.A. & Decl. & $z$ & $z$ Xue & $\begin{array}{l}\log L_{U V}{ }^{a} \\
\left(\operatorname{erg~s}^{-1}\right)\end{array}$ & $\begin{array}{c}\mathrm{SFR}^{\mathrm{b}} \\
\left(M_{\odot} \mathrm{yr}^{-1}\right)\end{array}$ & $\begin{array}{c}0.5-8 \mathrm{keV}^{\mathrm{c}} \\
\text { (counts) }\end{array}$ & $\begin{array}{c}\log L_{0.5-8}{ }^{\mathrm{d}} \\
\left(\mathrm{erg} \mathrm{s}^{-1}\right)\end{array}$ & $\Gamma$ & Notes ${ }^{\mathrm{e}}$ \\
\hline 344 & 53.10485 & -27.70521 & 1.5 & $1.6^{s}$ & 43.91 & 6.3 & $1811.3_{-48.7}^{+50.0}$ & $43.79 \pm 0.01$ & $2.0 \pm 0.1$ & L05a, R \\
\hline 405 & 53.12284 & -27.72279 & 1.9 & $1.6^{p}$ & 43.97 & 13.4 & $59.0_{-13.9}^{+15.1}$ & $42.93 \pm 0.11$ & $<0.1$ & $\mathrm{R}$ \\
\hline 308 & 53.09392 & -27.76774 & 1.9 & $1.7^{s}$ & 43.95 & 12.3 & $674.5_{-29.0}^{+30.2}$ & $43.92 \pm 0.02$ & $0.2 \pm 0.1$ & L05a \\
\hline 326 & 53.10082 & -27.71601 & 2.5 & $2.3^{s}$ & 44.58 & 54.9 & $234.4_{-20.5}^{+21.7}$ & $43.46 \pm 0.04$ & $1.6 \pm 0.2$ & $\mathrm{R}$ \\
\hline 137 & 53.03334 & -27.78257 & 3.0 & $2.6^{s}$ & 44.36 & 18.9 & $1249.4_{-39.2}^{+40.4}$ & $44.45 \pm 0.01$ & $1.3 \pm 0.1$ & $\mathrm{R}$ \\
\hline 490 & 53.14880 & -27.82112 & 3.0 & $2.6^{s}$ & 44.31 & 16.4 & $84.6_{-10.9}^{+12.1}$ & $43.43 \pm 0.06$ & $0.4 \pm 0.2$ & $\mathrm{R}$ \\
\hline $386^{\mathrm{f}}$ & 53.11792 & -27.73432 & 3.0 & $3.6^{p}$ & 44.38 & 20.1 & $37.4_{-10.6}^{+11.7}$ & $42.99 \pm 0.13$ & $>1.0$ & $\mathrm{R}$ \\
\hline 254 & 53.07600 & -27.87816 & 3.0 & $2.8^{s}$ & 44.67 & 44.1 & $246.2_{-19.4}^{+20.6}$ & $43.65 \pm 0.04$ & $1.8 \pm 0.2$ & L05a, L05 \\
\hline 563 & 53.17439 & -27.86735 & 3.0 & $3.6^{s}$ & 45.43 & 365.0 & $1389.1_{-41.4}^{+42.6}$ & $44.51 \pm 0.01$ & $1.4 \pm 0.1$ & L05a \\
\hline $573^{\mathrm{f}}$ & 53.17848 & -27.78403 & 3.0 & $3.2^{s}$ & 44.31 & 16.4 & $685.1_{-29.1}^{+30.3}$ & $44.14 \pm 0.02$ & $1.4 \pm 0.1$ & L05a, L05 \\
\hline 577 & 53.18015 & -27.82060 & 3.0 & $1.9^{s}$ & 45.41 & 342.6 & $2437.0_{-53.3}^{+54.5}$ & $44.67 \pm 0.01$ & $1.6 \pm 0.1$ & L05a, L05 \\
\hline 588 & 53.18464 & -27.88092 & 3.0 & $3.5^{s}$ & 44.80 & 64.0 & $322.4_{-23.1}^{+24.3}$ & $43.79 \pm 0.03$ & $1.8 \pm 0.2$ & L05a, L05 \\
\hline $388^{\mathrm{f}}$ & 53.11858 & -27.88478 & 3.8 & $3.0^{p}$ & 44.12 & 7.2 & $41.3_{-11.4}^{+12.7}$ & $43.38 \pm 0.13$ & $<0.3$ & $\ldots$ \\
\hline 374 & 53.11163 & -27.86078 & 3.8 & $3.7^{p}$ & 44.48 & 19.3 & $<26.5$ & $<43.11$ & $<0.7$ & $\ldots$ \\
\hline 262 & 53.07848 & -27.85984 & 3.8 & $3.7^{s}$ & 44.50 & 20.2 & $214.6_{-17.6}^{+18.9}$ & $44.19 \pm 0.04$ & $0.1 \pm 0.1$ & $\mathrm{R}$ \\
\hline $150^{\mathrm{f}}$ & 53.03989 & -27.79846 & 3.8 & $3.3^{p}$ & 44.11 & 6.9 & $38.6_{-10.6}^{+11.9}$ & $43.32 \pm 0.13$ & $<0.5$ & $\ldots$ \\
\hline $100^{f}$ & 53.01660 & -27.74484 & 3.8 & $3.9^{p}$ & 44.42 & 16.1 & $93.4_{-18.2}^{+19.3}$ & $43.63 \pm 0.09$ & $>1.1$ & $\ldots$ \\
\hline 546 & 53.16528 & -27.81405 & 3.8 & $3.1^{s}$ & 44.75 & 40.0 & $1231.0_{-38.7}^{+39.9}$ & $44.84 \pm 0.01$ & $0.4 \pm 0.04$ & L05a, L05, R \\
\hline 458 & 53.13850 & -27.82112 & 3.8 & $3.6^{p}$ & 44.49 & 19.8 & $27.6_{-6.7}^{+8.0}$ & $43.26 \pm 0.12$ & $0.2 \pm 0.04$ & $\ldots$ \\
\hline 371 & 53.11158 & -27.76777 & 5.0 & $3.1^{p}$ & 44.16 & 7.0 & $173.3_{-15.0}^{+16.3}$ & $44.19 \pm 0.04$ & $0.6_{-0.1}^{+0.2}$ & $\cdots$ \\
\hline
\end{tabular}

Notes.

a Observed (i.e., not dust-corrected) UV luminosity.

b Dust-corrected UV-derived SFR.

${ }^{c}$ Counts refer to the aperture-corrected (background-subtracted) net counts for the full band $(0.5-8 \mathrm{keV})$ from X11.

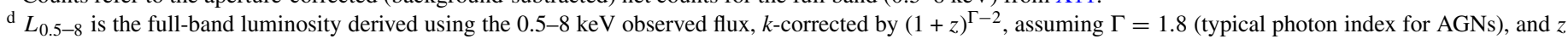

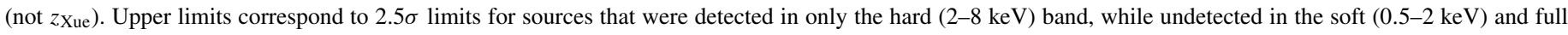
bands.

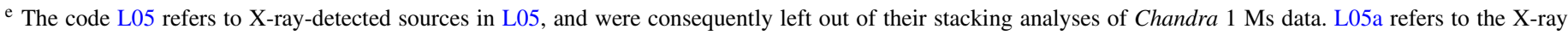
sources identified in the E-CDF-S catalog (Lehmer et al. 2005a). R indicates that this source was detected in the VLA radio data with S/N > 3 (see Section 2.5).

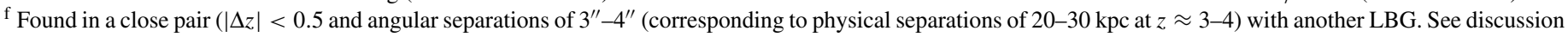
in the text (Section 3.1).

resampling. Bootstrap errors include the variation due to background fluctuations (Poisson error), therefore the uncertainties quoted in our analysis are the bootstrap errors.

To study the relationship between X-ray luminosity and SFR of $z \lesssim 4$ LBGs, we stack the LBG samples in two SFR bins: $\mathrm{SFR} / M_{\odot} \mathrm{yr}^{-1}=5-15$ and $\mathrm{SFR} / M_{\odot} \mathrm{yr}^{-1}=15-100$. For the remainder of our study, we require an $\mathrm{S} / \mathrm{N}$ cut of $2.5 \sigma$ for a stacked detection (which corresponds to $>99.38 \%$ confidence limit for one-sided Gaussian distributions). Since the low SFR samples do not yield any detections and suffer greatly from incompleteness, we do not include them in our discussion. The results of our stacking analyses are discussed in detail in Section 3.2.

\subsection{Contribution of AGNs to Average X-Ray Properties}

The 20 X-ray-detected sources in Table 3, which were classified as AGNs following the criteria outlined in Section 2.2, are useful for assessing the level of AGNs contamination in the stacked samples discussed in the previous section (Section 2.3).

In Figure 3, we show the cumulative AGN fractions as a function of X-ray luminosity for high SFR (15-100 $M_{\odot} \mathrm{yr}^{-1}$; filled blue symbols) and medium SFR (5-15 $M_{\odot} \mathrm{yr}^{-1}$; open green symbols) LBG samples. The different redshift samples are displayed with different symbols, as described in the legend.
The data are not sufficient to determine whether there is any variation in the AGN fraction with redshift. Focusing on the high SFR LBG sample (filled blue symbols), where we have some redshift information, there is no obvious redshift trend (e.g., the $z<3$ point appears consistent with the $z \approx 3.8$ data, while the $z \approx 3$ values are higher). We proceed by including high SFR data from $z<1.4$ star-forming galaxies from L08 (filled gray triangles). The fit from L08 (blue dashed line), using the mean SFR from the high SFR sample, compares well with our errorweighted fit (solid blue line) to all the data (L08 and our high SFR sample points). The fit from L08 (green dotted line; open gray triangles show medium SFR galaxies from this study), using the mean SFR from the medium SFR sample, appears consistent with our medium SFR fit (dotted green line) to data (open green circles).

Our higher redshift data probe a larger volume and extend the relation to higher $L_{\mathrm{X}}$. At lower $L_{\mathrm{X}}$, the difference between L08 may be from incompleteness at these lower X-ray luminosities. Therefore we estimate the AGN contribution from Equation (8) of L08 and subtract this from the stacked X-ray luminosity as described in L08. Since the AGN fractions from our analysis appear lower (or equal) to those from L08, we err on the side of overcompensating for the AGN contribution. Nevertheless, these corrections are smaller than the errors on $L_{X}$ (typically 


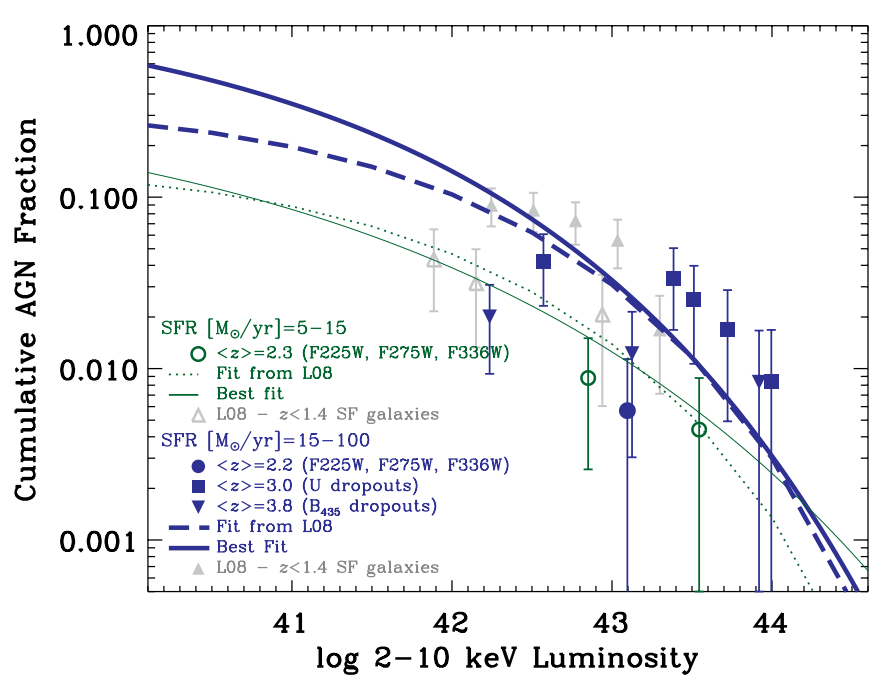

Figure 3. We show the cumulative AGN fraction vs. 2-10 keV X-ray luminosity for $\mathrm{SFR}=5-15 M_{\odot} \mathrm{yr}^{-1}$ (medium SFR; open green symbols) and $\mathrm{SFR}=$ 15-100 $M_{\odot} \mathrm{yr}^{-1}$ LBGs (high SFR; filled blue symbols) and for $z<1.4$ starforming galaxies from L08 (gray triangles; filled for high SFR and open for medium SFR). The different symbols describe different LBG samples, as listed in the legend. The solid lines mark the error-weighted exponential best fit to the combined data set for the high SFR (blue) and medium SFR (green) data; best fits determined from Equation (8) in L08, using mean SFRs from the full sample for high SFR and medium SFR galaxies are shown as blue dashed and green dotted lines, respectively.

(A color version of this figure is available in the online journal.)

$\sim 20 \%$ ). We note that L08 estimated $\sim 50 \%-70 \%$ AGN contamination in $z \approx 3$ LBGs, based on CDF-S 1 Ms analysis. Our increased depth, which improves identification and removal of AGNs, may account for the significant decrease in our measured AGN fraction; since low-luminosity AGNs are more numerous, survey depth does not necessarily scale proportionally with AGN fraction.

\subsection{Comparing Average SFRs Using Multiwavelength Data: Radio and IR SFRs}

We perform stacking of radio and IR observations to offer independent tests for estimating unattenuated SFRs for the $z<5$ LBG samples. Using VLA (N. Miller et al. 2013, in preparation; see also Miller et al. 2008) and PACS Hershel (Elbaz et al. 2011) data for the CDF-S, we have stacked all of the LBGs that are not X-ray detected. These measurements provide consistency checks between the radio-derived, UV + IR SFR, and the dustcorrected UV-derived SFRs.

We generated $50^{\prime \prime} \times 50^{\prime \prime}$ cutouts of the radio image around each LBG position, and proceeded to stack these cutouts for each LBG redshift sample. These LBG samples exclude the same galaxies as the $\mathrm{X}$-ray stacking sample (i.e., those sources that were individually X-ray detected or near, within twice the $99 \%$ EEF radius, other $\mathrm{X}$-ray-detected sources). Incidentally, 8 of the 20 individually X-ray-detected LBGs (listed in Table 3) were also individually detected (with $>3 \sigma$ ) in the VLA observations (marked " $\mathrm{R}$ " in the last column). Two stacking procedures were used: the first used a straight median for each pixel in the stack, and the second used an average with rejection of the highest and lowest pixel. The latter method is designed to prevent real sources within the $50^{\prime \prime}$ fields from seeping into the average and hence output stack image, which is more likely when the number of images to stack is large because of the surface density of such bright sources. These real sources are often extended radio emission from unrelated sources, although a small number of the LBGs themselves were individually detected ( 7 of the 13 radiodetected LBGs with radio $\mathrm{S} / \mathrm{N}>5 \sigma$ were also X-ray detected and are marked with "R" in the last column of Table 3 ) and the rejection prevented them from unduly influencing the resulting stack. For the $z \approx 3.8$ and $z \approx 5.0$ samples, the rejection was extended from the single highest pixel to the two highest pixels to ameliorate contributions of real detected sources and improve the cosmetics of the stacked image.

The stacked images were then inspected to evaluate the resulting rms noise level and any sources recovered by the stacking technique. The results of the stacks produced by the median and average procedures were always consistent in terms of the resulting rms noise and for the sample that yielded a weak detection. The rms of the stacks ranged from about $0.2 \mu \mathrm{Jy}$ to $1.3 \mu \mathrm{Jy}$ depending on the number of objects in the sample, down from the $\sim 6.4 \mu \mathrm{Jy}$ noise level typical of the radio image across the CDF-S area. Stacks made from cutouts using arbitrary positional offsets demonstrated that the rms noise values in the actual sample stacks were consistent, and these "blank sky" stacks produced no false detections. The $1.4 \mathrm{GHz}$ radio luminosities were $k$-corrected, assuming a standard power law with a spectral index of 0.7 , and converted to SFRs using the relation given in Yun et al. (2001, see Equation (13); using the Salpeter IMF with mass limits of 0.1 and $100 M_{\odot}$ ), but divided by a factor of 1.7 to translate SFRs to the Kroupa IMF.

Herschel-PACS data covering the GOODS-S (see Elbaz et al. 2011 for a description of the observations) was downloaded from the HeDaM Web site. ${ }^{20}$ For the purposes of this paper, we used only the $160 \mu \mathrm{m}$ data as, for our samples, it probes closer to the peak of the far-infrared spectral energy distribution (SED) than the other PACS wavebands and thus provides the most reliable indicator of the IR luminosity. To perform our stacking analyses, we extracted 60 pixel $\times 60$ pixel thumbnails (from both the science and rms images) centered on the positions of all the $\mathrm{X}$ ray undetected sources that are also undetected at $160 \mu \mathrm{m}$. For each of our redshift bins we combined corresponding science thumbnails using a weighted mean, weighting by the $1 / \sqrt{(\mathrm{rms})}$ value at each pixel position. We use aperture photometry to measure the flux within a circular aperture placed at the center of each stacked image. The error on the mean was calculated by randomly selecting (with replacement) two-thirds of each redshift sample, performing the stacking procedure 1000 times, then calculating the standard deviation of the resulting flux distribution. Finally, to determine the significance of each stacked (mean) flux, we compared it against that obtained when stacking the same number of random positions. The mean flux of the $160 \mu \mathrm{m}$ undetected sources was then combined with the mean flux of the detected sources using a weighted mean (weighted according to the number of undetected/detected sources) to give the mean flux of all sources in that bin (see Mullaney et al. 2012 for more details). We convert the stacked $160 \mu \mathrm{m}$ flux into a total IR $(8-1000 \mu \mathrm{m})$ luminosity using the template spectrum from Chary \& Elbaz (2001). Then, using Equation (3), where $L_{\mathrm{IR}}$ is the total IR luminosity, we calculate the UV + IR SFRs.

Table 4 summarizes the mean observed UV, dust-corrected $\mathrm{UV}$, radio and UV+IR SFRs. Only the $z=2.5 \mathrm{LBG}$ sample was detected in the radio stacking at $\sim 3 \sigma$; none of the LBG samples were detected in the IR stacking analysis. Within errors, the dust-corrected UV-derived SFRs are consistent with the radio and UV + IR SFRs.

\footnotetext{
${ }^{20}$ http://hedam.oamp.fr/GOODS-Herschel/goods-south_data.php
} 
Table 4

Comparing SFRs: UV, Radio, and IR

\begin{tabular}{lrcccc}
\hline \hline$z$ & $N$ & \multicolumn{4}{c}{$\langle$ SFR $\rangle\left(M_{\odot} \mathrm{yr}^{-1}\right)$} \\
\cline { 3 - 6 } & & $\mathrm{UV}$ & $\mathrm{UV}_{\text {corr }}$ & Radio & $\mathrm{UV}+$ IR \\
\hline 1.5 & 30 & $3_{-1}^{+2}$ & $11_{-5}^{+9}$ & $<27$ & $<25$ \\
1.9 & 44 & $3_{-1}^{+2}$ & $22_{-10}^{+17}$ & $<39$ & $<32$ \\
2.5 & 222 & $3_{-1}^{+2}$ & $11_{-6}^{+15}$ & $36 \pm 10$ & $<33$ \\
3.0 & 311 & $5_{-3}^{+6}$ & $16_{-9}^{+23}$ & $<45$ & $<48$ \\
3.8 & 1381 & $2_{-1}^{+3}$ & $5_{-3}^{+7}$ & $<30$ & $<61$ \\
5.0 & 701 & $3_{-2}^{+3}$ & $6_{-3}^{+8}$ & $<135$ & $<240$ \\
5.9 & 220 & $3_{-1}^{+2}$ & $4_{-2}^{+3}$ & $<304$ & $<590$ \\
6.8 & 61 & $2_{-1}^{+3}$ & $3_{-2}^{+6}$ & $<713$ & $<2200$ \\
8.0 & 44 & $2_{-1}^{+3}$ & $2_{-1}^{+3}$ & $<1064$ & $<4800$ \\
\hline
\end{tabular}

Notes. The mean UV, dust-corrected UV, radio and UV+IR SFRs for full sample of LBGs (only excluding individually detected X-ray sources and those sources within twice the $99 \%$ EEF radius from other X-ray sources).

\section{RESULTS AND DISCUSSION}

We divide our discussion of the results into three sections. The first section includes a brief summary of interesting discoveries related to individually detected LBGs from the 4 Ms CDF-S catalog (X11). The other sections relate to our stacking analyses. Based on our stacking results, we find that the $z \lesssim 4$ samples provide significant stacked detections, whereas we were only able to measure upper limits for the $z \gtrsim 5$ LBGs. Therefore, we consider it is most meaningful to split the stacking analysis into two parts: studying the evolution of the X-ray properties at $z \lesssim 4$ as related to XRBs and star formation, and constraining supermassive black hole (SMBH) growth for $z \approx 3-8$ LBGs.

\subsection{Individually Detected X-Ray Sources}

We use the probabilistic matches to optical/IR sources from the X11 catalog (see Section 2.2) to identify 20 LBGs, with redshifts ranging from $z=1.5-5.0$, that were individually detected in the Chandra 4 Ms data. The X11 catalog lists photometric or spectroscopic redshifts for all the sources (shown in Column 5 in Table 3). Therefore, the redshifts determined by the LBG dropout technique offer another measurement for comparison. The spectroscopic redshifts match those of the dropout technique reasonably well ( 8 of the 12 redshifts agree within $|\Delta z|<0.5)$. The four cases where the spectroscopic redshift differs significantly $(|\Delta z| \geqslant 0.5)$ from the dropout redshift include three $U$-dropouts $(z \approx 3.0 ;$ XIDs $=563,577$, and 588) and one $B_{435}$-dropout $(z \approx 3.8$; XID $=546)$, and in all these cases the spectroscopic redshift quality is "secure" $(\geqslant 95 \%$ confidence levels with multiple spectral features). With the exception of XID $=563$, all of these were detected in L05 and with the same dropout classification. While the discrepancies in values between the spectroscopic redshifts and the LBG selection seem considerably higher than the expected contamination rate (3\%-10\%, see Section 2), the redshift failure rates in these X-ray-detected LBGs are not representative of the LBG sample in general. In fact, these four AGNs are the most UV luminous of our entire sample, and therefore, most likely to contain high-ionization emission lines that are not modeled by the LBG selection.

The highest redshift LBG sample containing detected sources is $z=5$, with a single X-ray-detected source (XID $=371$ ). While the photometric redshift for this source in the X11 catalog

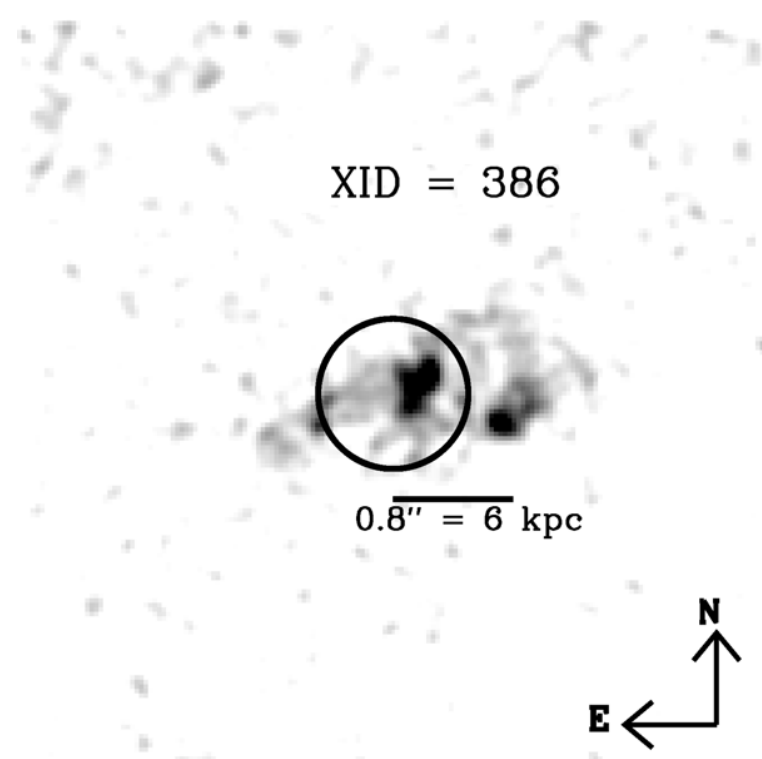

Figure 4. HST ACS $z 850$ image shows XID $=386$, an X-ray-detected LBG at $z \approx 3$, apparently interacting with another LBG (also at the same redshift). These LBGs have angular separations of $00^{\prime \prime} 8$, corresponding to a physical separation of $6 \mathrm{kpc}$.

is $z=3.1$, a more recent determination utilizing the CANDELS photometry gives a more likely redshift of $z=4.65_{-0.41}^{+0.18}$ (Xue et al. 2012).

We find that 5 of the $20 \mathrm{X}$-ray-detected LBGs appear to be in close pairs (with other LBGs, in the same redshift sample, within $30 \mathrm{kpc}$; marked with asterisks in Table 3). For comparison, 512 of the 4419LBGs $(\sim 12 \%)$ are in close pairs. Therefore, based on our crude estimate, X-ray-detected LBGs appear to have a higher probability (a factor of two enhancement) of being in a close pair than other LBGs. However, our analysis does not calculate the real space pair fraction (i.e., the width of the redshift bins, listed in Table 1, corresponds to large physical distances) and does not take into account the possibility of chance projections; therefore, we stress that a more detailed statistical analysis is required to measure accurate pair fractions and test the significance of close pair enhancement in X-ray-detected LBGs. In one case, $\mathrm{XID}=386$, the other LBG is separated by $0{ }^{\prime} 8$ (at $z=3$, this corresponds to a physical separation of $6 \mathrm{kpc}$; Figure 4). Inspecting the HST ACS $z_{850}$ image for this source, we observe two bright peaks, surrounded by diffuse extended emission. Given that the observed sizes of LBGs at $z \sim 3$ are typically smaller ( $\sim 2-3 \mathrm{kpc}$; Ferguson et al. 2004; Trujillo et al. 2006; Franx et al. 2008; Mosleh et al. 2011), it is unlikely that the LBGs belong to a single galaxy larger than $6 \mathrm{kpc}$. Rather, we speculate that these two separate galaxies are undergoing a merger. These sources also correspond to a detected radio source of $\sim 8 \sigma$. However, none of the other four close pairs were radio detections; in these cases, the angular separations are $3^{\prime \prime}-4^{\prime \prime}$, corresponding to physical separations $>20-30 \mathrm{kpc}$ at $z=3.0$ and 3.8. While further study of these pairs is beyond the scope of this paper, we note this potentially relevant observation and conjecture that the enhanced star formation and AGN activity could be related to interactions in these LBGs (see also Kocevski et al. 2012).

\subsection{X-Rays and Star Formation}

Concentrating on the X-ray properties related to star formation in LBGs, we split our five $z \lesssim 4$ LBG samples into two 


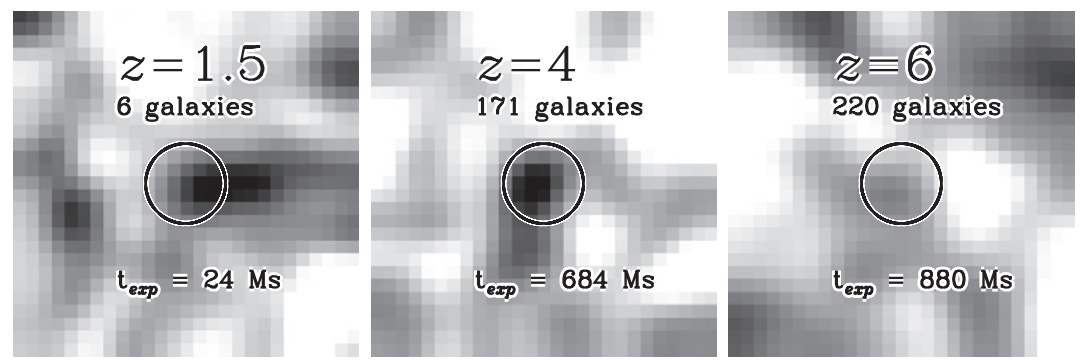

Figure 5. Observed $0.5-2 \mathrm{keV}$ stacked images for the $z \approx 1.5$ and $4 \mathrm{LBGs}$ with SFR $=15-100 M_{\odot} \mathrm{yr}^{-1}$ (left and middle) and $z=6$ (right) LBG samples are shown. These images have been smoothed by a 3 pixel kernel using Gaussian weighting. The $z=1.5$ image shows a $2.6 \sigma$ detection (near our detection threshold) and contains six galaxies ( $24 \mathrm{Ms}$ exposure), $z=4$ image shows a $4.0 \sigma$ detection and contains 171 galaxies, corresponding to 684 Ms of exposure. The $z=6$ image contains 220 galaxies ( $\sim 880 \mathrm{Ms}$ total exposure) with $\mathrm{S} / \mathrm{N}=1.0$. The marked circles have $3^{\prime \prime}$ radii.

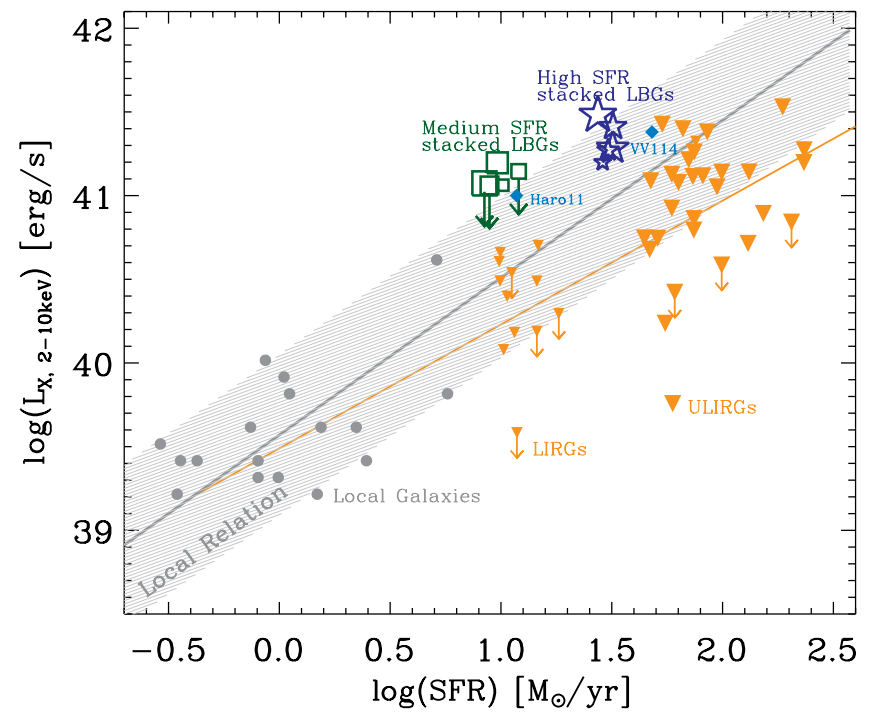

Figure 6. We compare the $2-10 \mathrm{keV}$ (rest-frame) luminosity vs. SFR for different galaxy populations to show that UV-selected galaxies appear to deviate toward higher $L_{X}$. Local galaxies (gray points from Colbert et al. 2004) and LIRGs/ULIRGs (orange triangles from Iwasawa et al. 2009; Lehmer et al. 2010). Stacked medium SFR LBGs (open green squares), stacked high SFR LBGs (open blue stars), two local analogs (cyan diamonds, Haro11; Grimes et al. 2007 and VV114; Grimes et al. 2006) all have higher X-ray luminosities per SFR compared to other galaxy populations. The LBG symbol sizes correspond to the redshifts, smallest $(z=1.5)$ to largest $(z=3.8)$. Solid gray line (shaded region) shows correlation (scatter) based on low SFR galaxies and solid orange line shows fit to data, including IR-selected galaxies, from Lehmer et al. (2010).

(A color version of this figure is available in the online journal.)

separate SFR bins for stacking: $\mathrm{SFR} /\left(M_{\odot} \mathrm{yr}^{-1}\right)=5-15$ (medium; see Figure 2 ) and $\mathrm{SFR} /\left(M_{\odot} \mathrm{yr}^{-1}\right)=15-100$ (high). We do not include the low SFR galaxies $\left(\mathrm{SFR}=1-5 M_{\odot} \mathrm{yr}^{-1}\right)$ in our analysis because the LBG samples suffer from incompleteness here (see Figure 2) and there are no stacked detections, with upper limits providing only weak constraints. Stacking of the high SFR LBGs all yield detections (with 2-10 keV X-ray luminosities ranging from 1.6-3.0 $\times 10^{41} \mathrm{erg} \mathrm{s}^{-1}$ ), while only the $z \approx 1.5$ and 3 samples are detected for the medium SFR bins. The results from this analysis are summarized in Table 5. Our least significant detection (at 2.6 $\sigma$ ) is the $z=1.5$ sample, whose stacked $0.5-2 \mathrm{keV}$ image is shown on the left side of Figure 5. Within each redshift and SFR bin, the mean and median values of the SFRs (given in Columns 6 and 7 of Table 2) agree fairly well, suggesting that the X-ray stacking results are representative for the whole sample and not skewed by galaxies in a particular SFR regime.

In Figure 6, we show the X-ray/SFR relation $\left(L_{\mathrm{X}}\right.$ versus SFR) for the stacked LBGs (open blue stars; sizes relate to redshift, smallest to largest range from $z=1.5-3.8$ ) as compared to other samples of star-forming galaxies: local galaxies from Colbert et al. (2004), luminous infrared and ultraluminous infrared galaxies (LIRGs/ULIRGs; orange triangles) from Lehmer et al. (2010) and Iwasawa et al. (2009). VV114 and Haro11 are two local LBG analogs (Grimes et al. 2006, 2007) and shown as labeled cyan diamonds in Figure 6. The stacked LBGs appear to have a similar X-ray/SFR ratio to the low SFR local galaxies (gray points). However, all of the UV-selected samples (the local analogs, VV114 and Haro11, and the stacked $z=1.5-4$ LBGs) appear to have slightly higher $L_{\mathrm{X}}$ per SFR. The gray line (and shaded region) follow the $L_{X}$ versus SFR relation (and $1 \sigma$ scatter) derived in Lehmer et al. (2010) for the gray points (hereafter, the local relation). The high SFR end of this relation is difficult to study since few local galaxies have such high SFRs. However, Lehmer et al. (2010) find that the infrared-selected galaxies (LIRGs/ULIRGs) deviate from the local relation, with lower $L_{X}$ per SFR (i.e., they are better fit by the orange solid line). They argue that in typical galaxies, both HMXBs and LMXBs provide substantial contributions to the total X-ray luminosity, while high specific SFR galaxies have $\mathrm{X}$-ray emission dominated by HMXBs alone, thereby causing a lower $L_{X} /$ SFR (see Equation (1)). Studying the X-ray/IR correlation in $0<z<2$ galaxies, Symeonidis et al. (2011) also find that the correlation deviates from the local correlation at high $L_{\mathrm{IR}}$, presumably due to relatively high specific SFRs. Other local $(z<0.2)$ samples of UV-selected galaxies, with high specific SFRs, can offer important insight at these high SFRs and further investigation of this relation at the high SFR end is ongoing (A. R. Basu-Zych et al. 2013, in preparation).

While UV-selected galaxies (locally and at $z>1.5$ ) appear similarly shifted in the X-ray/SFR relation compared to other galaxy populations, it has not been firmly established whether the local X-ray/SFR relation can be applied to high-redshift galaxies. Cowie et al. (2012) claim that the ratio of X-ray luminosity to UV luminosity does not evolve from the local relation, if you assume a constant dust attenuation factor (with values between 3 and 5) for stacked $z=1-6$ LBGs. In Figure 6, the stacked LBGs (open green squares and blue stars) show subtle variation with redshift (symbol size ranges from $z=1.5$, smallest, to $z=4$, largest). However, extending our analysis to $z<1.5$ star-forming galaxies and taking dust attenuation corrections into account, we further examine the X-ray/SFR relation for traces of evolution.

In Figure 7, we show the mean $L_{\mathrm{X}} / \mathrm{SFR}$ ratio of our sample and further include results from Laird et al. (2005, filled green diamond) for SFR $=5-15 M_{\odot} \mathrm{yr}^{-1}$, Laird et al. (2006, filled cyan diamond) for SFR $=15-100 M_{\odot} \mathrm{yr}^{-1}$, and Reddy et al. (2006, open purple diamonds) for SFR $>30 M_{\odot} \mathrm{yr}^{-1}$. 

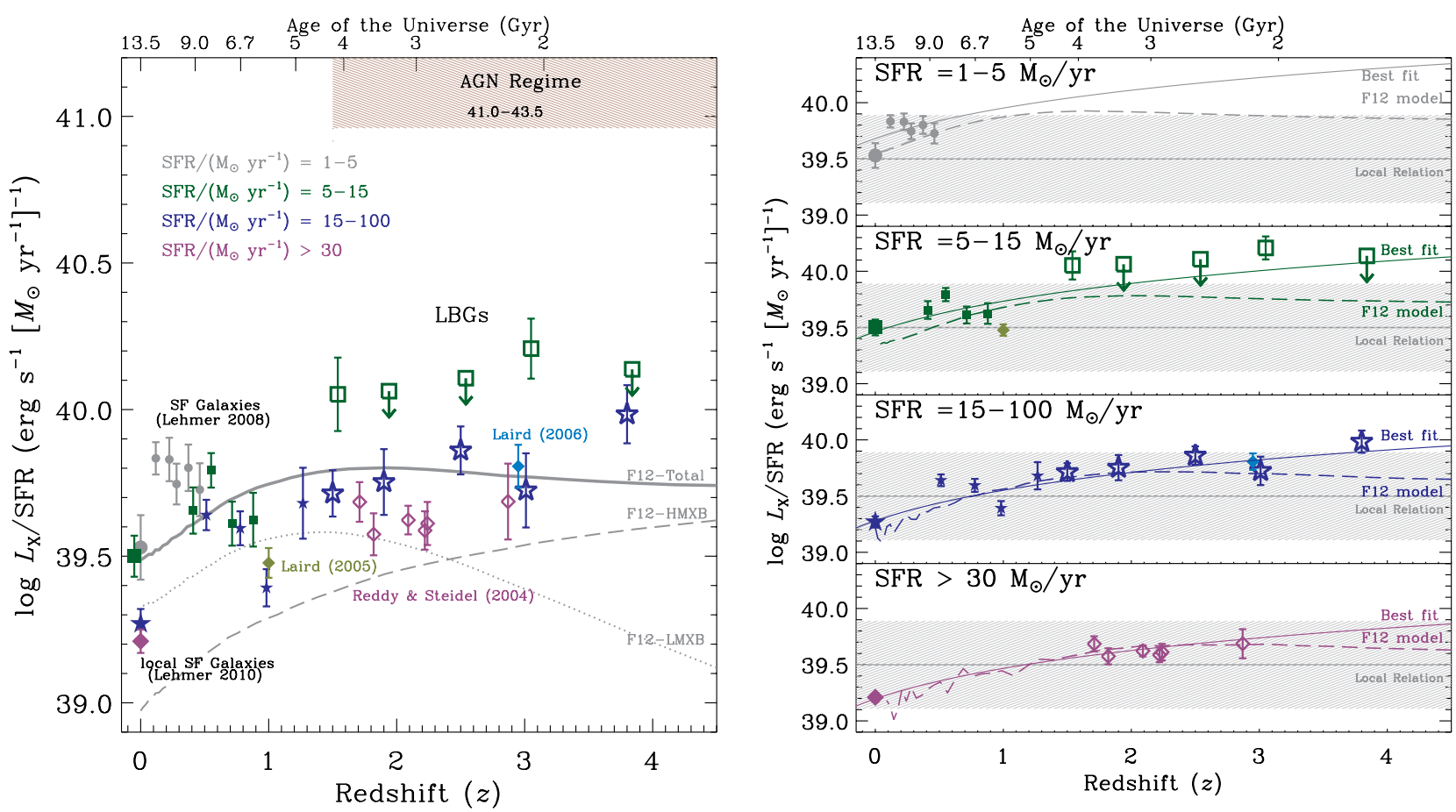

Figure 7. We show the evolution of rest-frame hard (2-10 keV) X-ray luminosity per SFR between $0<z<4$ (the last 12 Gyr of cosmic history). The left panel shows all the data, while the right panel separates the results into different SFR ranges to show redshift trends. Symbols mark bins of galaxies with different SFR ranges: gray circles have low SFRs, green squares show medium SFRs, blue stars show high SFR galaxies, and purple diamonds mark the highest SFR galaxies $\left(\mathrm{SFR} / M_{\odot} \mathrm{yr}^{-1}>\right.$ 30). Following the same scheme, larger filled symbols show local $(z \sim 0$; Lehmer et al. 2010) galaxies, and smaller filled symbols are star-forming galaxies between $0<z<1.4$ (L08). Stacking results from other star-forming galaxies are shown: $z=1$ Balmer break galaxies (filled green diamond; Laird et al. 2005), $z=1.5-3$ BX/BM galaxies (open purple diamonds; Reddy \& Steidel 2004), and $z=3$ LBGs (filled cyan diamond; Laird et al. 2006). Left: the red hatched region shows the regime, $\log L_{\mathrm{X}}=41.0-43.5 \mathrm{erg} \mathrm{s}^{-1}$, inhabited by individually detected X-ray sources, assumed to be AGNs. The gray curves show X-ray binary synthesis models from F12 for total (LMXB + HMXBs; solid), HMXBs (dashed), and LMXBs (dotted) for galaxies with SFRs $>1 M_{\odot} \mathrm{yr}^{-1}$. Right: the gray line and shaded region (shown in all panels) represent the local X-ray/SFR relation and its scatter (derived by L08). The best-fit model parameterization (solid curves) shows weak redshift evolution, as described by Equation (6). X-ray binary synthesis models from F12 (dashed curves) are not fits to the data and show excellent agreement with the data and our best fit.

(A color version of this figure is available in the online journal.)

Table 5

Summary of Stacked X-Ray/Star Formation Properties in $z<4$ LBGs

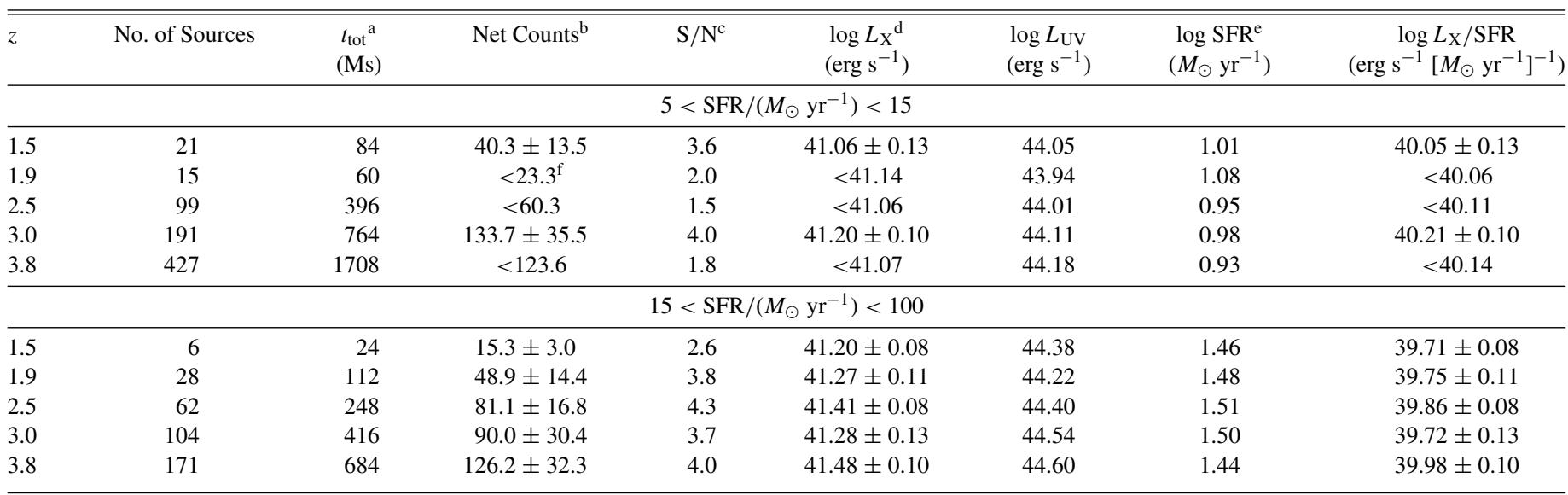

Notes.

a Total exposure time of the stack.

${ }^{b}$ Background-subtracted counts in observed 0.5-2 keV band. Errors include Poisson and bootstrap errors (as described in Section 2.3).

c $\mathrm{S} / \mathrm{N}$ is measured as $S / \sqrt{B}$, where $S$ and $B$ are net and background counts, respectively.

d Rest-frame 2-10 keV luminosity, $k$-corrected from observed $0.5-2 \mathrm{keV}$. See the text for more details.

e Dust-corrected SFR.

${ }^{\mathrm{f}}$ Upper limits are $2.5 \sigma$ values.

For comparison with lower redshift samples, we include the local star-forming galaxies from Lehmer et al. (2010) and the $0<z<1.4$ star-forming galaxies from L08. We note that the high SFR point at $z=0$ from Lehmer et al. (2010) is dominated by IR-selected galaxies (as discussed previously, these appear to have lower $L_{\mathrm{X}}$ per SFR).

Combining the information from the data shown in Figure 7, including our LBGs sampled by redshift and SFR bins (given 


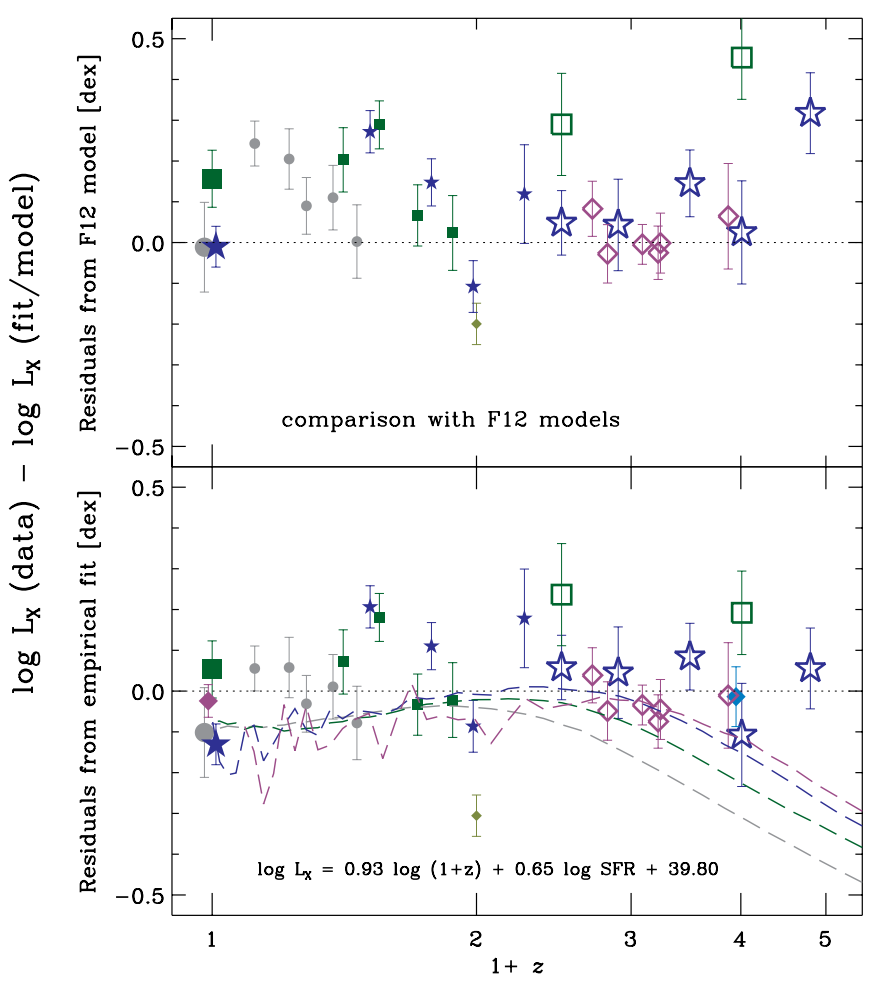

Figure 8. We compare residuals between data and X-ray binary synthesis models from F12 (top panel) and empirical fits (bottom panel) vs. redshift. The colors and symbols are as described for Figure 7. Top panel: F12 models agree excellently with the data at all redshifts but best for the low SFR and high SFR samples at $z=0$ and the SFR $>30 M_{\odot} \mathrm{yr}^{-1}$ at $z>2.5$. Bottom panel: we display the residuals of the empirical fit ( $\log L_{X}$ is fit by the equation stated here) to the data (symbols) and F12 models (dashed curves; with the colors corresponding to the SFR bins as in the previous figure: gray, green, blue, and purple represent SFR $/ M_{\odot} \mathrm{yr}^{-1}=1-5,5-15,15-100$, and $>30$, respectively). (A color version of this figure is available in the online journal.)

in Table 5) and other published data, we parameterize the X-ray luminosity in terms of redshift and SFR. Using error-weighted least-squares fitting to the stacked detections, we derive the following best fit to the available data:

$$
\begin{aligned}
\log L_{\mathrm{X}} & =A \log (1+z)+B \log \mathrm{SFR}+C \\
A & =0.93 \pm 0.07 \\
B & =0.65 \pm 0.03 \\
C & =39.80 \pm 0.03 .
\end{aligned}
$$

The trend for increasing $L_{\mathrm{X}} / \mathrm{SFR}$ with decreasing $\mathrm{SFR}$ is consistent with what is observed for local galaxies. For example, Lehmer et al. (2010) find $L_{X}$ varies with SFR in a similar way (within uncertainties, referring to coefficient $B$ in their Table 4) for their local sample of LIRGs.

In addition, we find evidence of weak redshift evolution (shown as the solid curve in the right panel of Figure 7, where SFR in the equation is set to the mean SFR of the displayed sample). Our result (Equation (6)) is robust even when we restrict our analysis to the most complete samples (high SFR LBGs), where selection effects (i.e., flux limits) are minimal. Therefore, we argue that this evolution is driven by that of physical properties (e.g., metallicity, star formation history) within the galaxies.

F12 perform a large-scale population synthesis study, using the StarTrack binary population synthesis code (Belczynski et al. 2002, 2008), which models XRB populations from the first galaxies of the universe until today. They use as input to their

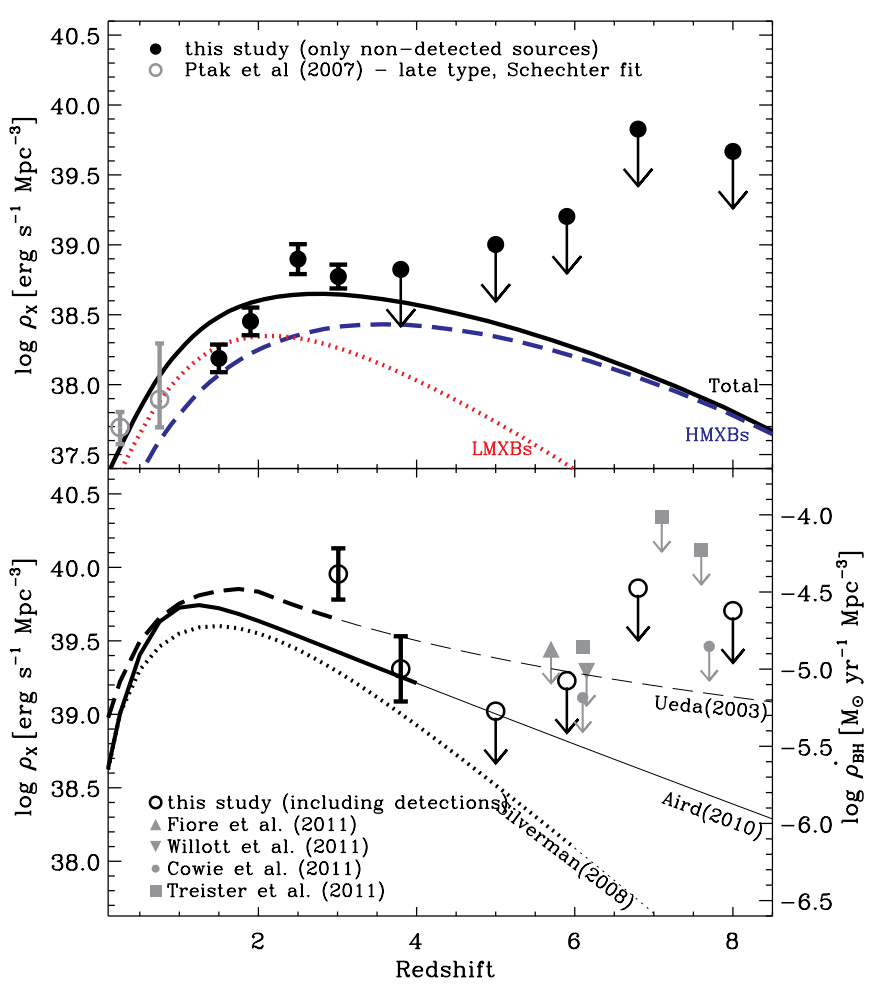

Figure 9. Top: the observed evolution of the normal-galaxy XLD, $\rho_{X}$, from our stacked LBGs (solid black points) and $z<1.4$ late-type galaxy X-ray luminosity function (open gray circles; Ptak et al. 2007) shows excellent agreement with XRB models (solid black, dashed blue and red dotted lines for total, HMXBs and LMXBs, respectively; for SFR $>1 M_{\odot} \mathrm{yr}^{-1}$ galaxies). Bottom: open circles show total $\rho_{\mathrm{X}}$ (including individually detected sources) from stacking, which are primarily dominated by X-ray emission from AGNs. Results from other stacking analyses are shown in gray symbols, as described in the legend. The curves show X-ray luminosity function models, as labeled, where thinner lines show extrapolated values. The axis on the right side provides black hole mass accretion rates (see the text for details). Since no individually detected LBGs were present in the $z \gtrsim 6$ sample, the solid (top panel) and open (bottom panel) black circles are identical at these redshifts.

(A color version of this figure is available in the online journal.)

modeling the Millennium II Cosmological Simulation (BoylanKolchin et al. 2009) and the updated semi-analytic galaxy catalog by Guo et al. (2011) to self-consistently account for the star formation history and metallicity evolution of the universe. Their models, which are constrained by the observed X-ray properties of local galaxies, give predictions about the global scaling of emission from XRB populations with properties such as SFR and stellar mass, and the evolution of these relations with redshift.

The results presented in F12 correspond to a general population of galaxies, including a mixture of actively star-forming and passive early type galaxies. In this paper, we consider the maximum likelihood model, as reported in F12, which we adapt to the galaxy sample properties of our survey. More specifically, while F12 used all the galaxies in the Millennium II simulation box in order to derive the star formation history and metallicity evolution, here we only took into account the evolution of galaxies with SFR in four bins $\left(1-5,5-15,15-100,>30 M_{\odot} \mathrm{yr}^{-1}\right)$. We assume that LBGs are representative of the galaxy population over these redshifts $(z=1-5)$ and therefore any metallicity and star formation history evolution in the LBGs will also be described by galaxies in the Millennium II simulation (and therefore, in the F12 models). These adapted models can be directly compared to our stacking results (see Figures 7-9). 
Noting that these XRB synthesis models are not fits to our data, there is remarkable agreement of these models with the data and empirical fits. The top panel of Figure 8 shows the residuals between the F12 models and data versus redshift. Residuals between the empirical fit and the data are shown in the bottom panel of Figure 8. The dashed curves show the differences between this best-fit relationship and the F12 models, with the colors corresponding to SFR bins as in the previous figure: gray, green, blue, and purple represent $\mathrm{SFR} /\left(M_{\odot} \mathrm{yr}^{-1}\right)=1-5,5-15$, $15-100$, and $>30$, respectively.

We use the F12 models to interpret the evolution in the $\mathrm{X}$-ray/SFR relation. The evolution of 2-10 $\mathrm{keV}$ emission per SFR from XRBs involves several competing effects: star formation history, the evolution of XRB populations, and metallicity evolution. The contributions of HMXBs and LMXBs shift with the global star formation history of the universe; HMXBs trace the young stellar populations and are expected to scale with SFR, while LMXBs track older stellar populations that dominate once the young stars are extinct. This effect can be seen in the shape of the LMXB curve (dotted gray) in Figure 7 (left), which includes the global star formation history. Metallicity affects the number of HMXBs and their $\mathrm{X}$-ray luminosities; high mass stars, which eventually evolve into black hole HMXBs (more X-ray luminous than neutron star $\mathrm{HMXBs}$ ), are easier to produce in high metallicity environments because of weaker stellar winds (F12). At higher redshifts, metallicities are lower and the contribution of HMXBs to the $\mathrm{X}$-ray luminosity is higher for a given SFR, apparent from the gray dashed line (HMXB) in Figure 7 (left). The combination of these effects on the X-ray luminosity per SFR is shown as the solid gray line in left panel of Figure 7 . With additional information (e.g., accurate stellar masses, metallicities, and star formation histories), we could further explore how well the XRB synthesis models describe these separate effects in observed galaxies. However, in the absence of such data, we interpret the evolution of X-ray luminosity per SFR to be driven by the metallicity evolution of HMXBs. Given their high sSFRs $\left(10^{-8.7}-10^{-8.0}\right.$, consistent with containing young stellar populations), HMXBs are likely to dominate the X-ray emission in LBGs; metallicity evolution in HMXBs causes an increase in the $L_{\mathrm{X}} / \mathrm{SFR}$ with redshift. We further note that this result, the mild evolution of $L_{X} / S F R$ with redshift, seems consistent with the constraints that Dijkstra et al. (2012) determined based on the cosmic X-ray background.

\subsection{Evolution of X-Ray Luminosity Density as Related to X-Ray Binaries}

To determine the total X-ray contribution of XRBs in the universe, we stack the full samples of LBGs (including all SFRs, but excluding X-ray-detected sources). The top panel of Figure 9 provides our measurements of the $2-10 \mathrm{keV} X$-ray luminosity density (XLD), $\rho_{\mathrm{X}}$, due to star formation from LBGs. $\rho_{\mathrm{X}}$ was calculated in the following way:

$$
\begin{aligned}
\rho_{\mathrm{x}} & =n_{\mathrm{LBG}}\left\langle L_{\mathrm{X}}\right\rangle \\
& =\left\langle L_{X}\right\rangle \int_{L_{\mathrm{min}}}^{\infty} \phi\left(L_{\mathrm{UV}}\right) d L_{\mathrm{UV}}
\end{aligned}
$$

by multiplying the average (stacked) rest-frame $2-10 \mathrm{keV} X$-ray luminosity, $\left\langle L_{\mathrm{X}}\right\rangle$, by the LBG number density (by integrating the $\mathrm{LBG}$ luminosity function, $\phi\left(L_{\mathrm{UV}}\right)$, down to the faintest observed UV magnitude limit for the sample; refer to papers listed in Table 1 for luminosity function fitting parameters). We also include $\rho_{\mathrm{X}}$ values determined from the X-ray luminosity function, fit by a Schechter function, of late-type, low redshift $(z<1.4$ ), galaxies (Ptak et al. 2007, converted from $0.5-2 \mathrm{keV}$ band into $2-10 \mathrm{keV}$ using a power-law SED with $\Gamma=2$ ), shown as gray open circles. We compare the measured values to those predicted by F12 based on total XRB contribution (black) and separated into contributions from HMXBs (dashed blue) and LMXBs (dotted red). In general, there is good agreement of the total XRB curve with the data; the models include all galaxies with SFRs $>1 M_{\odot} \mathrm{yr}^{-1}$, while the minimum SFR varies slightly between redshift samples in the data (see Figure 2). For example, at $z \approx 1.5$, the measured value appears slightly lower than the expected $\rho_{X}$ from HMXBs + LMXBs, which may be caused by incompleteness in the $z \approx 1.5 \mathrm{LBG}$ sample. Another explanation is that $z=1.5 \mathrm{LBG}$ may not be representative of all high SFR ( $>1 M_{\odot} \mathrm{yr}^{-1}$ galaxies; i.e., the LBG selection would miss dusty star-forming galaxies like LIRGs or low sSFR galaxies dominated by older stellar populations). The $z \approx 2.5$ measured value appears slightly higher than the model, and may include contamination from low luminosity AGN.

\section{4. $z \approx 3-8$ Constraints on the Evolution of Supermassive Black Holes}

At $z \gtrsim 5$, we are unable to obtain detections by stacking only sources that are individually X-ray undetected. However, by including individually detected X-ray sources, we can provide constraints on black hole growth in these galaxies. Table 6 summarizes the results from our $z \gtrsim 5$ stacking. The top section of Table 6 excludes individually detected X-ray sources, while the bottom section lists the results from stacking all the sources. We note that including X-ray-detected sources in our stacking analysis at $z \gtrsim 5$ only affects the measurement at $z \approx 5$ (slightly, because of one X-ray-detected source). Therefore, while the results at $z \gtrsim 6$ remain unchanged, we shift our focus from studying accretion in XRBs to that in SMBHs.

In the bottom panel of Figure 9, we show our measurements of the $2-10 \mathrm{keV}$ XLD, $\rho_{\mathrm{X}}$, from LBGs. $\rho_{\mathrm{X}}$ was calculated by multiplying the average (stacked) X-ray luminosity, $\left\langle L_{X}\right\rangle$, by the number density (as described in Section 3.2 for the top panel of this figure). Here, the X-ray luminosities have been calculated assuming $\Gamma=1.8$, which is appropriate for AGNs (whereas, all of our previous calculations have been using $\Gamma=1.7$, see Section 2.3). This observed quantity, $\rho_{\mathrm{X}}$, can be used to estimate a black hole mass accretion rate density $\left(\rho_{\mathrm{BH}}\right.$, shown in log units on the axis at right side) for the universe by assuming a black hole radiation efficiency $(\epsilon)$ and bolometric correction $\left(C_{\text {bol }}\right)$ as

$$
\dot{\rho}_{\mathrm{BH}}=\frac{(1-\epsilon)}{\epsilon c^{2}} C_{\mathrm{bol}} \rho_{\mathrm{X}} M_{\odot} \mathrm{yr}^{-1} \mathrm{Mpc}^{-3},
$$

where $c$ is the speed of light, $\rho_{\mathrm{X}}$ is given on the axis on left, and we adopt the values $\epsilon=0.1$ and $C_{\text {bol }}=25$ following Treister et al. (2010). In this figure, we show the total (including the single individually detected source, listed in Table 3) stacked $\mathrm{X}$-ray luminosity per $\mathrm{Mpc}^{-3}$ as open black circles. To compare with other studies, we use the $0.5-2 \mathrm{keV}$ count rates ${ }^{21}$ from other high redshift stacking studies to convert consistently to X-ray luminosities as described in Section 2.3; these results are shown as the gray upward triangle (Fiore et al. 2012a), downward

\footnotetext{
21 Where $3 \sigma$ upper limits are given, we converted to $2.5 \sigma$ upper limits to the count rates for consistency with our analysis.
} 
Table 6

Properties of Stacked $1.5<z<8$ LBGs Related to X-Ray Luminosity Density

\begin{tabular}{|c|c|c|c|c|c|c|c|}
\hline$z$ & No. of Sources & $\begin{array}{c}t_{\mathrm{tot}} \\
(\mathrm{Ms})\end{array}$ & Net Counts & $\mathrm{S} / \mathrm{N}$ & $\begin{array}{l}\log L_{X}{ }^{a} \\
\left(\operatorname{erg~s}^{-1}\right)\end{array}$ & $\begin{array}{l}\log L_{\mathrm{UV}} \\
\left(\mathrm{erg} \mathrm{s}^{-1}\right)\end{array}$ & $\begin{array}{c}\log \rho_{\mathrm{X}} \mathrm{b} \\
\left(\mathrm{erg} \mathrm{s}^{-1} \mathrm{Mpc}^{-3}\right)\end{array}$ \\
\hline \multicolumn{8}{|c|}{ Total X-ray from X-ray binaries (No SFR cut; excluding individually detected X-ray sources ${ }^{\mathrm{c}}$ ) } \\
\hline 1.5 & 30 & 120.0 & $56.26 \pm 14.36$ & 4.2 & 41.06 & 44.12 & $38.19 \pm 0.10$ \\
\hline 1.9 & 44 & 176.0 & $69.16 \pm 17.69$ & 4.3 & 41.20 & 44.17 & $38.45 \pm 0.10$ \\
\hline 2.5 & 222 & 888.0 & $117.68 \pm 32.89$ & 3.3 & 41.01 & 44.15 & $38.90 \pm 0.11$ \\
\hline 3.0 & 311 & 1244.0 & $226.38 \pm 49.08$ & 5.4 & 41.21 & 44.50 & $38.77 \pm 0.09$ \\
\hline 3.8 & 1381 & 5524.0 & $<222.48$ & 1.6 & $<40.82$ & 44.12 & $<38.82$ \\
\hline 5.0 & 701 & 2804.0 & $<158.51$ & 0.9 & $<41.18$ & 44.18 & $<39.00$ \\
\hline \multicolumn{8}{|c|}{ Total X-ray from all sources (including individually detected X-ray sources ${ }^{\mathrm{d}}$ ) } \\
\hline 3.0 & 319 & 1276.0 & $3513.55 \pm 1736.9$ & 82.1 & 42.39 & 44.52 & $39.96 \pm 0.17$ \\
\hline 3.8 & 1388 & 5552.0 & $667.99 \pm 445.7$ & 7.5 & 41.31 & 44.12 & $39.31 \pm 0.22$ \\
\hline 5.0 & 702 & 2808.0 & $<158.62$ & 2.0 & $<41.20$ & 44.18 & $<39.02$ \\
\hline 5.9 & 220 & 880.0 & $<88.56$ & 1.0 & $<41.62$ & 44.10 & $<39.23$ \\
\hline 6.8 & 61 & 244.0 & $<47.07$ & -1.0 & $<41.98$ & 44.18 & $<39.86$ \\
\hline 8.0 & 44 & 176.0 & $<40.03$ & -1.8 & $<42.20$ & 44.12 & $<39.71$ \\
\hline
\end{tabular}

Notes.

a Total luminosity in $2-10 \mathrm{keV}$ (rest-frame) band.

b Total luminosity density in $2-10 \mathrm{keV}$ (rest-frame) band.

${ }^{c}$ Since $z=5.9,6.8$, and 8.0 have no individually detected X-ray sources, the information for these redshifts is the same as given below.

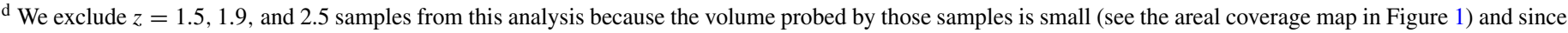

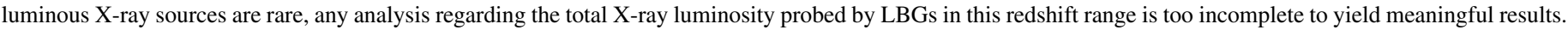

triangle (Willott 2011), small circle (Cowie et al. 2012), and square symbols (Treister et al. 2010).

We note these other studies have more sources in their stack since they limit sources with off-axis angles greater than $9^{\prime}$ versus our adopted $7^{\prime}$ off-axis limit; however, we have checked that using the $9^{\prime}$ limit does not change the results of our stacking analysis by much $(<10 \%)$. In agreement with Fiore et al. (2012a), Cowie et al. (2012), and Willott (2011), we do not obtain a detection for the stacked $z \approx 5.9$ sample (see the right image in Figure 5), which was previously reported in Treister et al. (2010). Rather our upper limits for the $z \approx 6$ and 7 data are very similar to those of Fiore et al. (2012a). Willott (2011) uses an optimized aperture and weighting in order to push their upper limits slightly lower than the others. ${ }^{22}$ At $z \approx 6$, our upper limit is lower than the $5 \sigma$ detection claimed by Treister et al. (2010, though this detection has been called into question in other papers; see Willott 2011; Cowie et al. 2012). The solid, dashed, and dotted lines show the 2-10 keV XLD evolution model from Aird et al. (2010), Ueda et al. (2003), and Silverman et al. (2008), extrapolated beyond the measured redshifts (at $z=4,3.0$, and 6.0, respectively; displayed in thinner lines) assuming the same form and derived parameters. Our observations are most consistent with the XLD evolution of AGNs with $L_{X}>10^{42} \mathrm{erg} \mathrm{s}^{-1}$ as predicted by Aird et al. (2010) and less consistent (based on the upper limits at $z \approx 5-6$ ) with Ueda et al. (2003). Yet, we are not able to definitively rule out the Ueda et al. (2003) models since extrapolation to higher redshifts $(z>3)$ may not be accurate, and at $z=3$ the observations are consistent with their model. Additionally, calculating $\rho_{\mathrm{X}}$ from AGNs in LBGs alone may underestimate the XLD from the entire AGN population, present also in populations of galaxies not selected by the LBG selection (e.g., submillimeter galaxies). We may be underestimating the contributions from

22 Since Fiore et al. (2012a) and Willott (2011) do not include CANDELS LBGs, their upper limits are not as constrained as ours. But when we exclude the CANDELS data, our upper limits are the same as theirs.
Compton-thick AGN: Gilli et al. (2011) detect a Comptonthick AGN at $z=4.76$, suggesting that Compton-thick AGN may be important (though difficult to detect and study) for understanding black hole evolution in the early universe.

\section{SUMMARY AND FUTURE WORK}

Using the deepest X-ray survey to date, the CDF-S 4 Ms data, and the most comprehensive LBG catalogs, we have stacked $1.5<z<8$ LBGs. We have split our analysis into two separate redshift ranges: $z \lesssim 4$ to study the relation between SFR and $\mathrm{X}$-rays produced by XRBs, and $z \gtrsim 5$ to place constraints on the black hole accretion history of the universe. Our main results are as follows.

1. We expand the list of X-ray detections (found in the X11 catalog) associated with LBGs (see Table 3), based on probabilistic matching between X-ray and optical/infrared catalogs. Within $7^{\prime}$ of the Chandra pointing, we match 20 LBGs with individually detected X-ray sources, assumed to be AGNs based on their X-ray luminosities $\left(>10^{42} \mathrm{erg} \mathrm{s}^{-1}\right)$.

2. While the local X-ray/SFR relation does appear to apply to the high-redshift LBGs, it also has significant scatter. We find that the population of UV-selected galaxies (including local LBG analogs, also selected by using the rest-frame UV) preferentially lie at the high end of the X-ray/SFR correlation scatter (see Figure 6). The high SFR regime of this relation has not been well studied for different samples of galaxies, but these observations deviate from what is observed for high SFR, IR-selected LIRGs, and ULIRGs (Lehmer et al. 2010; Symeonidis et al. 2011).

3. We discover mild evolution of $L_{\mathrm{X}}$ per SFR between $0<$ $z<4$ (see Figure 7). We stacked the LBGs in two different SFR ranges: 5-15 $M_{\odot} \mathrm{yr}^{-1}$ (medium) and 15-100 $M_{\odot} \mathrm{yr}^{-1}$ (high). We obtain $>2.5 \sigma$ detections in the stacked high SFR bins, and for two bins $(z=1.5$ and 3$)$ in the stacked medium SFR samples. We compare our results for the high SFR galaxies with XRB population synthesis 
models (F12) and find excellent agreement (see Figure 8). We find that the rest-frame $2-10 \mathrm{keV}$ X-ray luminosity relates to SFR and redshift in the following way: $\log L_{\mathrm{X}}=$ $0.93 \log (1+z)+0.65 \log \mathrm{SFR}+39.8$.

4. We calculate the total (including all SFRs, but excluding individually detected X-ray sources) XLD for different redshift samples to compare with XRB population synthesis models (F12, see the top panel of Figure 9). We find that HMXBs and total (HMXBs +LMXBs) are both consistent with the data.

5. Stacking $z \gtrsim 5$ LBGs did not provide any detections (see the bottom panel of Figure 9). However, we use the upper limits to derive the 2-10 keV XLD and we compare with results from other studies and models (Aird et al. 2010; Silverman et al. 2008; Ueda et al. 2003). Our results are similar to Fiore et al. (2012a), Cowie et al. (2012), and Willott (2011): we do not detect LBGs at $z=6$ (contrary to Treister et al. 2010). Our observations are consistent with the models from Aird et al. (2010) and Silverman et al. (2008), extrapolated to $z=8$; our upper limit at $z \sim 5$ lies below the extrapolated $(z>3)$ Ueda et al. (2003) model. However, we note that our comparisons with XLD evolution models are subject to the following caveats: extrapolation of the XLDs beyond the redshifts for which they were measured may not be accurate; studying AGNs in LBGs may not account for the complete AGN population at these redshifts (e.g., AGNs in submillimeter galaxies); we may be underestimating the contribution from Compton-thick AGN.

Future missions like the James Webb Space Telescope (JWST) aim to discover the first galaxies, when the universe was only a few 100 million years old, searching for very high redshift $(z>10)$ LBGs. Unfortunately, studying such distant LBGs in the X-rays would be impossible with current X-ray telescopes, and X-ray instruments capable of such ambitious ventures exist only the imagination, as yet. However, the future does offer other opportunities for studying X-ray emission from distant LBGs.

Fiore et al. (2012b) describe the future prospects for studying AGNs in LBGs using future X-ray facilities like Athena and Super-Chandra. As for studying XRB populations in these galaxies, deeper X-ray observations would add stacked detections (where we now have upper limits) and better constrain the results presented in this paper. We estimate, using our Equation (6), that a $5 \mathrm{Ms}$ Chandra exposure (i.e., adding $1 \mathrm{Ms}$ to the current CDF-S observations) would provide stacked detections $(>3 \sigma)$ for all the $z \approx 2.5-3.8$ medium SFR sample, and 6-8 Ms Chandra exposure would provide a stacked detection for the 100 highest SFR LBGs at $z \approx 5$. Deep IR observations from JWST and Herschel would provide additional rest-frame optical and IR photometry to provide better redshift determinations for the current LBGs at $z=5-8$, improving the purity of the LBG selection and offering more accurate measurements of stellar masses, dust attenuations, star formation histories, and SFRs for individual LBGs. X-ray stacking by these properties would provide insight about how the evolution of fundamental properties with redshift relate to the evolution of X-ray emission in these galaxies.

We are grateful to the Chandra Director's office for commissioning the $4 \mathrm{Ms}$ observation of the CDF-S. We thank Ezequiel Treister for helpful discussions and the anonymous referee for suggestions. This research was supported by Chandra Cycle 12 program No. 12620841 (PI: Basu-Zych) and NASA
ADP Proposal 09-ADP09-0071 (PI: Hornschemeier). A.R.B. gratefully acknowledges the appointment to the NASA Postdoctoral Program at the Goddard Space Flight Center, administered by Oak Ridge Associated Universities through a contract with NASA, and NASA's Swift Observatory for salary support. We gratefully acknowledge financial support from the Einstein Fellowship Program (B.D.L.), the Youth 1000 Plan program and the USTC startup funding (Y.Q.X.). P.O. acknowledges support provided by NASA through Hubble Fellowship grant HF-51278.01. T.F. acknowledges support from the CfA and the ITC prize fellowship programs. W.N.B., B.L., and Y.Q.X. thank CXC grant SP1-12007A and NASA ADP grant NNX10AC99G. Herschel is an ESA space observatory with science instruments provided by European-led Principal Investigator consortia and with important participation from NASA. The GOODS-H data were accessed through the HeDaM database (http://hedam.oamp.fr) operated by CeSAM and hosted by the Laboratoire d'Astrophysique de Marseille.

\section{REFERENCES}

Aird, J., Nandra, K., Laird, E. S., et al. 2010, MNRAS, 401, 2531 Alexander, D. M., Bauer, F. E., Brandt, W. N., et al. 2003, AJ, 126, 539 Alexander, D. M., Bauer, F. E., Chapman, S. C., et al. 2005, ApJ, 632, 736 Beckwith, S. V. W., Stiavelli, M., Koekemoer, A. M., et al. 2006, AJ, 132, 1729 Belczynski, K., Kalogera, V., \& Bulik, T. 2002, ApJ, 572, 407

Belczynski, K., Kalogera, V., Rasio, F. A., et al. 2008, ApJS, 174, 223 Bell, E. F., Papovich, C., Wolf, C., et al. 2005, ApJ, 625, 23 Blain, A. W., Smail, I., Ivison, R. J., \& Kneib, J.-P. 1999, MNRAS, 302, 632 Bouwens, R. J., Illingworth, G. D., Blakeslee, J. P., \& Franx, M. 2006, ApJ, 653,53

Bouwens, R. J., Illingworth, G. D., Franx, M., \& Ford, H. 2007, ApJ, 670, 928 Bouwens, R. J., Illingworth, G. D., Franx, M., \& Ford, H. 2008, ApJ, 686, 230 Bouwens, R. J., Illingworth, G. D., Franx, M., et al. 2009, ApJ, 705, 936 Bouwens, R. J., Illingworth, G. D., Oesch, P. A., et al. 2010, ApJ, 709, L133 Bouwens, R. J., Illingworth, G. D., Oesch, P. A., et al. 2011, ApJ, 737, 90 Bouwens, R. J., Illingworth, G. D., Oesch, P. A., et al. 2012a, ApJ, 752, L5 Bouwens, R. J., et al. 2012b, ApJ, 754, 83

Boylan-Kolchin, M., Springel, V., White, S. D. M., Jenkins, A., \& Lemson, G. 2009, MNRAS, 398, 1150

Brandt, W. N., Hornschemeier, A. E., Alexander, D. M., et al. 2001, AJ, 122, 1 Broos, P. S., Getman, K. V., Povich, M. S., et al. 2011, ApJS, 194, 4

Bunker, A. J., Wilkins, S., Ellis, R. S., et al. 2010, MNRAS, 409, 855 Chary, R., \& Elbaz, D. 2001, ApJ, 556, 562

Colbert, E. J. M., Heckman, T. M., Ptak, A. F., Strickland, D. K., \& Weaver, K. A. 2004, ApJ, 602, 231

Cowie, L. L., Barger, A. J., \& Hasinger, G. 2012, ApJ, 748, 12 Dijkstra, M., Gilfanov, M., Loeb, A., \& Sunyaev, R. 2012, MNRAS, 421, 213

Efron, B. 1982, The Jackknife, the Bootstrap and Other Resampling Plans (CBMS-NSF Regional Conference Series in Applied Mathematics; Philadelphia: SIAM)

Elbaz, D., Dickinson, M., Hwang, H. S., et al. 2011, A\&A, 533, A119 Fabbiano, G. 1989, ARA\&A, 27, 87

Ferguson, H. C., Dickinson, M., Giavalisco, M., et al. 2004, ApJ, 600, L107

Fiore, F., Puccetti, S., Grazian, A., et al. 2012a, A\&A, 537, A16

Fiore, F., Puccetti, S., \& Mathur, S. 2012b, Adv. Astron., 2012, doi: $10.1155 / 2012 / 271502$

Fragos, T., et al. 2012, arXiv:1206.2395

Franx, M., van Dokkum, P. G., Schreiber, N. M. F., et al. 2008, ApJ, 688, 770

Gilli, R., Norman, C., Vignali, C., et al. 2011, ApJ, 730, L28

Grimes, J. P., Heckman, T., Hoopes, C., et al. 2006, ApJ, 648, 310

Grimes, J. P., Heckman, T., Strickland, D., et al. 2007, ApJ, 668, 891

Grogin, N. A., Kocevski, D. D., Faber, S. M., et al. 2011, ApJS, 197, 35

Guo, Q., White, S., Boylan-Kolchin, M., et al. 2011, MNRAS, 413, 101

Hathi, N. P., Ryan, R. E., Jr., Cohen, S. H., et al. 2010, ApJ, 720, 1708

Hornschemeier, A. E., Brandt, W. N., Alexander, D. M., et al. 2002, ApJ, 568,82

Iwasawa, K., Sanders, D. B., Evans, A. S., et al. 2009, ApJ, 695, L103

Kennicutt, R. C. 1998, ARA\&A, 36, 189

Kocevski, D. D., Faber, S. M., Mozena, M., et al. 2012, ApJ, 744, 148

Koekemoer, A. M., Faber, S. M., Ferguson, H. C., et al. 2011, ApJS, 197, 36

Kroupa, P. 2001, MNRAS, 322, 231 
Laird, E. S., Nandra, K., Adelberger, K. L., Steidel, C. C., \& Reddy, N. A. 2005, MNRAS, 359, 47

Laird, E. S., Nandra, K., Hobbs, A., \& Steidel, C. C. 2006, MNRAS, 373,217

Laird, E. S., Nandra, K., Pope, A., \& Scott, D. 2010, MNRAS, 401, 2763

Lee, K.-S., Giavalisco, M., Gnedin, O. Y., et al. 2006, ApJ, 642, 63

Lehmer, B. D., Alexander, D. M., Bauer, F. E., et al. 2010, ApJ, 724, 559

Lehmer, B. D., Brandt, W. N., Alexander, D. M., et al. 2005a, ApJS, 161, 21

Lehmer, B. D., Brandt, W. N., Alexander, D. M., et al. 2005b, AJ, 129, 1 (L05)

Lehmer, B. D., Brandt, W. N., Alexander, D. M., et al. 2007, ApJ, 657, 681

Lehmer, B. D., Brandt, W. N., Alexander, D. M., et al. 2008, ApJ, 681, 1163 (L08)

Lehmer, B. D., Xue, Y. Q., Brandt, W. N., et al. 2012, ApJ, 752, 46

Madau, P., Ferguson, H. C., Dickinson, M. E., et al. 1996, MNRAS, 283, 1388

Meurer, G. R., Heckman, T. M., \& Calzetti, D. 1999, ApJ, 521, 64

Miller, N. A., Fomalont, E. B., Kellermann, K. I., et al. 2008, ApJS, 179, 114

Mineo, S., Gilfanov, M., \& Sunyaev, R. 2012, MNRAS, 419, 2095

Mosleh, M., Williams, R. J., Franx, M., \& Kriek, M. 2011, ApJ, 727, 5

Mullaney, J. R., Pannella, M., Daddi, E., et al. 2012, MNRAS, 419, 95

Oesch, P. A., Bouwens, R. J., Carollo, C. M., et al. 2010a, ApJ, 725, L150

Oesch, P. A., Bouwens, R. J., Illingworth, G. D., et al. 2010b, ApJ, 709, L16

Oesch, P. A., Bouwens, R. J., Illingworth, G. D., et al. 2012, ApJ, 745, 110

Persic, M., \& Rephaeli, Y. 2007, A\&A, 463, 481

Ptak, A., Griffiths, R., White, N., \& Ghosh, P. 2001, ApJ, 559, L91
Ptak, A., Mobasher, B., Hornschemeier, A., Bauer, F., \& Norman, C. 2007, ApJ 667,826

Ptak, A., Serlemitsos, P., Yaqoob, T., \& Mushotzky, R. 1999, ApJS, 120, 179

Ranalli, P., Comastri, A., \& Setti, G. 2003, A\&A, 399, 39

Reddy, N. A., \& Steidel, C. C. 2004, ApJ, 603, L13

Reddy, N. A., \& Steidel, C. C. 2009, ApJ, 692, 778

Reddy, N. A., Steidel, C. C., Erb, D. K., Shapley, A. E., \& Pettini, M. 2006, ApJ, 653,1004

Silverman, J. D., Green, P. J., Barkhouse, W. A., et al. 2008, ApJ, 679, 118

Stark, A. A., Gammie, C. F., Wilson, R. W., et al. 1992, ApJS, 79, 77

Steidel, C. C., Adelberger, K. L., Shapley, A. E., et al. 2000, ApJ, 532, 170

Steidel, C. C., \& Hamilton, D. 1992, AJ, 104, 941

Steidel, C. C., \& Hamilton, D. 1993, AJ, 105, 2017

Steidel, C. C., Pettini, M., \& Hamilton, D. 1995, AJ, 110, 2519

Symeonidis, M., Georgakakis, A., Seymour, N., et al. 2011, MNRAS, 417, 2239

Thompson, R. I., Illingworth, G., Bouwens, R., et al. 2005, AJ, 130, 1

Treister, E., Schawinski, K., Volonteri, M., et al. 2010, Natur, 474, 356

Trujillo, I., Förster Schreiber, N. M., Rudnick, G., et al. 2006, ApJ, 650, 18

Ueda, Y., Akiyama, M., Ohta, K., \& Miyaji, T. 2003, ApJ, 598, 886

Watson, C. R., Kochanek, C. S., Forman, W. R., et al. 2009, ApJ, 696, 2206

Willott, C. J. 2011, ApJ, 742, L8

Xue, Y. Q., Luo, B., Brandt, W. N., et al. 2011, ApJS, 195, 10 (X11)

Xue, Q., Wang, S. X., Brandt, W. N., et al. 2012, ApJ, 758, 129

Yun, M. S., Reddy, N. A., \& Condon, J. J. 2001, ApJ, 554, 803 